\title{
A simple paradigm for active and nonlinear microrheology
}

\author{
Todd M. Squires \\ Department of Physics and Department of Applied \& Computational Mathematics, \\ California Institute of Technology, Pasadena, California 91125 \\ John F. Brady \\ Department of Chemical Engineering, California Institute of Technology, Pasadena, California 91125
}

(Received 12 November 2004; accepted 23 May 2005; published online 1 July 2005)

In microrheology, elastic and viscous moduli are obtained from measurements of the fluctuating thermal motion of embedded colloidal probes. In such experiments, the probe motion is passive and reflects the near-equilibrium (linear response) properties of the surrounding medium. By actively pulling the probe through the material, further information about material properties can be obtained, analogous to large-amplitude measurements in (macro-) rheology. We consider a simple model of such systems: a colloidal probe pulled through a suspension of neutrally buoyant bath colloids. We choose a system with hard-sphere interactions but neglect hydrodynamic interactions, which is simple enough to permit analytic solutions, but nontrivial enough to raise issues important for the interpretation of experiments in active and nonlinear microrheology. We calculate the microstructural deformation for arbitrary probe size and pulling rate (expressed as a dimensionless Péclet number $\mathrm{Pe}$ ). From this, we determine the average retarding effect on the probe due to the microstructure, as well as fluctuations about this average. The high- $P e$ limit is singular, giving a finite Brownian contribution even in the limit of negligible diffusion. Significantly, different results are obtained for probes driven at constant velocity and constant force. Furthermore, we demonstrate that a probe pulled with an optical tweezer (roughly a harmonic well) can behave as fixed-force, fixed-velocity, or as a mixture of those modes, depending on the strength of the trap and on the pulling speed. More generally, we discuss how these results relate to previous work on the rheology of colloidal suspensions. Not surprisingly, the present theory (which ignores hydrodynamic interactions) gives shear thinning but no shear thickening; we expect that the incorporation of hydrodynamics would result in shear thickening as well. The effective micro- and macro-viscosities, when appropriately scaled, are in semi-quantitative agreement. This seems remarkable, given the rather significant difference in the two methods of measurement. However, for more complicated or unknown materials, where such scaling relations may not be known in advance, the comparison between micro- and macro may not be so favorable, which raises important questions about the relation between micro- and macrorheology. Finally, by analogy with previous work on macrorheology, we propose methods to scale up the present (dilute) theory to account for more concentrated suspensions, and suggest new active microrheological experiments to probe different aspects of suspension behavior. (C) 2005 American Institute of Physics. [DOI: 10.1063/1.1960607]

\section{INTRODUCTION}

While traditional materials like solids, liquids, and gases have been studied for centuries, the study of "complex" fluids has emerged relatively recently. ${ }^{1}$ This is surprising, given their ubiquity in biology, industry, food science, and personal care products. Broadly speaking, traditional schemes classify materials in terms of solids or fluids based on their equilibrium phase behavior derived from energy arguments; dynamics are omitted altogether. On the other hand, the rich variety of behavior exhibited by complex fluids results directly from dynamic effects. Silly putty is a canonical example: it flows like a viscous liquid if left to sit for several minutes, but bounces like an elastic solid in response to a fast impact. This illustrates a property of fundamental importance to complex fluids: their behavior depends on the time scale of interrogation.

Rheology is the study of the deformation and flow of materials, and rheological measurements of material properties are typically performed over a range of time scales. Measured features in the frequency-dependent viscous ("loss") and elastic ("storage") moduli belie microstructural processes that, in turn, determine the macro-scale behavior of the material. For example, the long entangled polymers that comprise silly putty do not have time to relax in response to a rapid shock and give a high-frequency elastic behavior, whereas they can relax via reptation and allow the material to flow like a viscous fluid if forced over a long time scale.

Traditional rheological measurements probe material properties by shearing a macroscopic volume of the sample between two solid surfaces of given geometry, such as the cone and plate. A small-amplitude, oscillitory shearing motion is typically applied, so that the material is perturbed only slightly from equilibrium, and its linear response is measured. Because of the weakly nonequilibrium nature of these measurements, powerful relations can be derived concerning 
the linear viscoelastic regime. ${ }^{1}$ Nonlinear rheological measurements can also be performed by significantly straining the material, which enables additional information to be obtained, such as shear thinning and shear thickening, ${ }^{2,3}$ normal stress differences, ${ }^{4}$ and relaxation processes. ${ }^{5-9}$

However, some materials of interest (particularly biomaterials) are difficult to procure in the large quantities required for traditional rheometers. To address such issues, techniques encompassing "microrheology" are being developed using small colloidal beads as tracers. ${ }^{10-14}$ The most straightforward technique records the positions of the colloids as they fluctuate due to Brownian motion. More sophisticated "two-point" techniques enable measurements of heterogeneous materials by measuring and cross-correlating the fluctuating motion of two well-separated tracer beads. ${ }^{15} \mathrm{In}$ both cases, the tracers move passively within the material, and the material is assumed to maintain a near-equilibrium configuration. This allows the use of the fluctuationdissipation theorem from statistical mechanics and the "generalized Stokes-Einstein-Sutherland relation" (GSESR) ${ }^{16}$ to relate the fluctuating positions directly to the viscous and elastic moduli of the material. As such, "passive" microrheological experiments are expected to mirror the linearresponse macrorheological measurements, ${ }^{14,17}$ although questions remain about, e.g., the role of specific interactions between the probe and the background material. ${ }^{18,19}$

Forcing and flows in the real world, however, need not be so gentle. It is therefore of interest to explore and understand the behavior of materials driven beyond the linearresponse regime. Techniques in "active" microrheology, in which a probe colloid is externally forced to move through a material, are currently under investigation using magnetic ${ }^{20-25}$ or optical forces. ${ }^{26,27}$ Active experiments allow the nonlinear response of the material to be tested, and an effective viscosity to be inferred from the relationship between driving force and probe velocity. In such cases, the microstructure itself can be deformed significantly, so that the material response differs from the linear response case, and the fluctuation-dissipation theorem and GSESR relation do not hold. Furthermore, confocal microscopy allows the perturbed microstructure itself to be visualized, providing an experimental window into the relationship between the nonlinear microstructural deformations (which can be measured directly), and the resulting "macroscopic" response of the material.

Many interesting and important questions are raised in these active and nonlinear microrheological experiments. Most importantly, what exactly is being measured? What connections, if any, do the quantities measured in these experiments have with those obtained using conventional macrorheology? How general are the measurements-how do they depend on the size and properties of the probe itself? How important are fluctuations in these measurements, and how do they scale? Conventional macrorheology employs two driving modes: constant shear stress and constant strain rate. By analogy, is there a distinction between active microrheological probes driven at constant velocity and constant force? Which mode (if either) is employed in active microrheology, and what difference does it make? What ad- ditional information might be provided by active microrheology that is inaccessible to its passive analog, or over conventional rheology?

Because microrheology remains in the early stages of development, it faces important and reasonable questions concerning its veracity, and ultimately its utility. Currently, the analogous macrorheological measurements serve as the benchmark against which microrheologically measured properties are compared. After all, agreement between the two techniques would certainly inspire confidence in the microrheological results; furthermore, one could immediately map the machinery developed for macrorheology onto microrheological measurements. However, it is worth asking whether such agreement is necessary for microrheology to be considered worthwhile. After all, both macro- and microrheology are concerned with probing complex material properties; any disagreement between the two would indicate that different information is encoded in the different measurements, and more (not less) information would be learned. In such a case, one could not use the macrorheological machinery to analyze the microrheological data; however, since the data itself are microrheological, it would seem fitting to try to understand it using microrheological models. Macro- and microrheology probe different aspects of the material: the former makes measurements over extremely long (macroscopic) length scales using a viscometric flow field, whereas the latter effectively measures material properties on the scale of the probe itself (since flow and deformation fields decay on this length scale). As the probe increases in size, one might expect that since the length scales over which the material is probed become sufficiently long that micro- and macrorheology would agree. However, it may very well be that they do not agree-even in the continuum (large-probe) limit - one measurement uses a viscometric flow, the other does not. A final, but important, point: materials that cannot be produced or procured in quantities sufficient for macrorheology (i.e., those materials that originally motivated microrheology) would not allow such agreement to be checked.

In this article, we propose and examine a simple model system for active and nonlinear microrheology. This model is simple enough to allow transparent analysis and to admit analytical solutions, yet sufficiently nontrivial that it contains the physics essential toward an understanding of many features generic to active and nonlinear microrheology. In particular, we demonstrate that even this simplest of model systems yields insight into many of the questions raised above. After describing the model colloidal system (Sec. II) and the role of microstructural perturbations (Sec. III), we derive an equation for microstructural dynamics (Sec. IV). Initially, we solve for the steady-state microstructural deformation in two simple limiting cases and the "average" apparent viscosity that results (Sec. V), as well as fluctuations about that average (Sec. VI). We then solve for the microstructural perturbation for arbitrary Péclet number (ratio of the external forcing to the thermal restoring forces) and present viscosity and fluctuation results for general $\mathrm{Pe}$ (Sec. VII). We examine the difference between constant-force and constant-velocity driving modes (Sec. VIII), and raise the possibility that current experiments may, in fact, mix these modes of operation. 
In Sec. IX we make a direct comparison between micro- and macrorheology for the simple model considered here, and (by analogy) suggest how the dilute results can be scaled up to higher concentrations and to include hydrodynamic interactions. Last, since our model is meant to be simple yet illustrative, we discuss its shortcomings, possible generalizations and connections to techniques such as falling-ball viscometry (Sec. X).

Finally, we note that microrheological experiments can provide an opportunity to rigorously examine the theoretical framework used to understand rheological properties of colloidal suspensions. The approach we adopt here is among the simplest variations of this framework. We make certain approximations that may seem overly simplistic, yet which have yielded remarkably accurate results in other contexts. It would be of significant value and interest to determine whether this prior success was simply fortuitous, or whether, in fact, such approximations and the accompanying physical interpretation may be more generally applied. Active and nonlinear microrheology, with its wealth of data, provides a promising platform for such studies.

\section{MODEL SYSTEM}

Complex fluids encompass a wide variety of materials and exhibit diverse properties. Certain key attributes, however, are shared among them, including a microstructure that gives rise to the "complex" macroscale material behavior. The microstructure can be driven out of equilibrium and "heals" by thermal motion, so that the (time-dependent) microstructural deformations give rise to a material response that is time-scale and amplitude dependent. Here, we examine perhaps the simplest model complex fluid to exhibit these traits: a suspension of neutrally buoyant rigid colloidal spheres.

We consider the behavior of a microrheological "probe" colloid particle in a suspension of "bath" particles. In passive microrheology, the probe fluctuates due to Brownian motion; in active microrheology, the probe is pulled through the material (Fig. 1). Various forces can exist between the probe and bath particles, including electric/osmotic forces between charge double layers, van der Waals forces, steric repulsions arising from grafted polymer "hairs," and hard-core repulsions between the solid spheres themselves. ${ }^{28}$ To model these interactions in the simplest way, we adopt a hard-sphere potential,

$$
\begin{aligned}
& U(r<a+b)=\infty, \\
& U(r>a+b)=0,
\end{aligned}
$$

so that the colloids do not interact until their hard-sphere radii touch, whereupon a force is exerted to prevent the hardsphere radii $a$ and $b$ of the probe and bath particles, respectively, from overlapping. (Appendix A explores the more general case.)

One might naturally assume the hard-sphere radii $a$ and $b$ to be the same as the physical "hydrodynamic" radii $a_{h}$ and $b_{h}$ where the no-slip boundary condition is obeyed. However, colloidal forces (e.g., electrostatic and steric) are often ex-

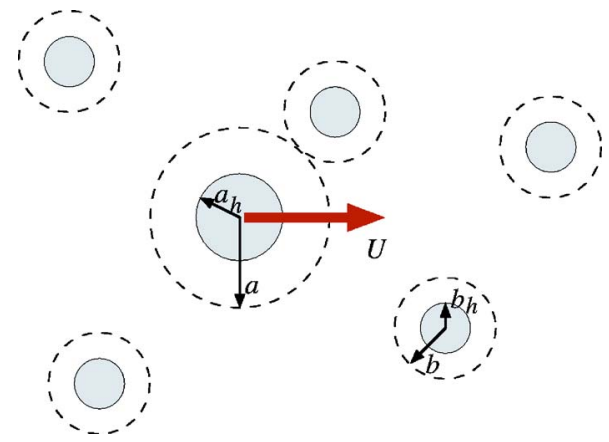

FIG. 1. Model system for active and nonlinear microrheology. A probe particle of hydrodynamic radius $a_{h}$ is pulled at velocity $U$ (or with force $F$ ) through a suspension of neutrally buoyant bath particles of hydrodynamic radius $b_{h}$. Long-range repulsive interactions are modeled with a hard-sphere potential at effective radii of $a=\lambda a_{h}$ and $b=\lambda b_{h}$, respectively. In principle, this model system can be used to treat any size ratio $a / b$. In practice, however, the neglect of hydrodynamics is only valid when $\lambda \gg 1$, which only occurs when the extended repulsive interactions between the probe and bath particles occur over a length scale that is large with respect to their hydrodynamic radii. The present results are most reliable when $\lambda$ is of order 1 .

erted beyond the physical boundary of the colloid, and particles can "collide" without their surfaces touching. In general, hydrodynamic radii are smaller than hard-sphere radii, and the ratios $\lambda_{a}=a / a_{h}$ and $\lambda_{b}=b / b_{h}$ exceed 1 . This forms the basis for the "excluded annulus" model, in which a parameter $\lambda$ "tunes" hydrodynamic interactions: ${ }^{29}$ hydrodynamic interactions between particles are negligible as $\lambda$ $\rightarrow \infty$, become increasingly important as $\lambda$ decreases and include near-body "lubrication" interactions as $\lambda \rightarrow 1$. The distance $a-a_{h}$ reflects the physics of the interaction, and is thus related to, e.g., the ionic screening length or the length of grafted polymer "hairs." In practice, the physics of the interactions will limit $\lambda$, particularly for large particles. This limitation should be kept in mind throughout this work, as we will consider arbitrary probe to bath particle size ratio,

$$
\alpha=\frac{a}{b},
$$

where $0<\alpha<\infty$. In the large-probe $(\alpha \gg 1)$ limit, the range of the interactions may not be long enough for the neglect of hydrodynamic interactions to be accurate.

In the present work, for simplicity, we neglect hydrodynamic interactions $(\lambda \rightarrow \infty)$. Although this may seem to be a severe approximation, it allows a straightforward and transparent analysis that captures and illustrates many of the significant physics of active, nonlinear microrheological experiments. Furthermore, this limit has been surprisingly effective in reproducing experimental measurements of the shear viscosity of colloidal suspensions, for which all particles are equivalent $(a=b) .{ }^{30-33}$ Remarkably, the effective shear viscosity derived from the infinite $\lambda$ limit does not differ appreciably from the results for $\lambda$ as small as 1.1. (Khair and Brady find this also holds for the microviscosity when $a=b .^{34}$ ) For even smaller $\lambda$, lubrication forces give rise to shear thickening at high shear rates. Experimentally, such behavior is seen as well: the onset of shear thickening can be delayed significantly when colloids are kept apart with electrostatic charges ${ }^{2}$ or grafted polymer "hairs". 35 In the shear 
viscosity problem, then, nonlubrication hydrodynamic interactions play only a quantitative role in the low- $P e$ limit, and the excluded annulus model captures the behavior quite well. An important question concerns to what extent this behavior is general, to which we shall return in Sec. IX. In general, our results should hold whenever the "hard-sphere" radii are much larger than the physical, or hydrodynamic radii. This effectively places an upper limit on the particle size (probe or bath) for which this theory can be expected to hold. How effective the theory will be for more extreme values of $\alpha$ remains to be seen.

\section{AVERAGE PROBE MOTION}

We now turn to the motion of the probe particle and the dynamics of the suspension itself. Because deformations to the microstructure are of central importance to the rheological response (micro or macro) of the material, we must solve for the distribution of bath particles surrounding the moving probe. The stochastic nature of Brownian motion requires such distributions to be found statistically. We denote the probability density for finding the probe and bath particles in a given configuration as $P_{N}\left(\boldsymbol{x}_{1}, \boldsymbol{x}_{2}, \ldots, \boldsymbol{x}_{N}\right)$, where the label 1 denotes the probe particle and $2 \rightarrow N$ the bath particles, and $\boldsymbol{x}_{\boldsymbol{\xi}}$ denotes the position of particle $\boldsymbol{\xi}$. (Particle momentum is not important for colloids.) Particles in suspension move under the action of external forces (the probe) and entropic Brownian forces (probe and bath). In the absence of hydrodynamic interactions $(\lambda \rightarrow \infty)$ the velocity of the probe particle is given by

$$
\boldsymbol{U}=M\left[\boldsymbol{F}^{\mathrm{ext}}-k_{B} T \nabla \ln P_{N}\right],
$$

where the mobility of the probe $M=1 / 6 \pi \eta a_{h}$ and we have dropped the label 1 denoting the probe particle for clarity. The viscosity of the suspending fluid is $\eta, k_{B} T$ is the thermal energy and $\boldsymbol{F}^{\text {ext }}$ denotes the external force moving the probe particle.

As shown in Appendix A, averaging (4) over the positions of the $N-1$ bath particles and integrating by parts results in an exact formula for the average probe velocity:

$$
\langle\boldsymbol{U}\rangle=M\left[\boldsymbol{F}^{\mathrm{ext}}-n k_{B} T \oint_{r=a+b} \boldsymbol{n} g(\boldsymbol{r}) d S\right] .
$$

In (5) the integral is over the surface of "contact" between the probe and bath particles, $r=a+b$, with normal $\boldsymbol{n}$ directed radially out from the probe. The number density of bath particles is $n$, and $g(\boldsymbol{r})$ is the pair-distribution function: the probability density for finding a bath particle at $\boldsymbol{r}$ relative to the probe particle. Equation (5) was derived for a constant imposed external force $\boldsymbol{F}^{\text {ext }}$; for a constant imposed velocity the formula is the same (in the absence of hydrodynamic interactions) with the average now about the external force rather than the velocity. While equation (5) is the same for both cases, the pair-distribution function $g(\boldsymbol{r})$ is not, as will become evident.

From (5) we see clearly that the thermal motion of the bath particles acts to resist the motion of the probe particle: there is a buildup of bath particles in front of the probe particle, and a deficit or wake trailing it, resulting in a reac- tive force that slows the probe (see Fig. 5). In the absence of an external force on the probe (i.e., in equilibrium), $g(\boldsymbol{r})$ is isotropic and the integral in (5) is zero. Near equilibrium-in the linear response regime- the departure from equilibrium is proportional to the external force and the average velocity is linear in $\boldsymbol{F}^{\mathrm{ext}}$,

$$
\langle\boldsymbol{U}\rangle=M_{\infty}^{s} \boldsymbol{F}^{\mathrm{ext}},
$$

where $k_{B} T M_{\infty}^{s} \equiv D_{\infty}^{s}$ is the long-time self-diffusivity of the probe particle in the surrounding bath particles (see Sec. IX and Appendix A). This connection between diffusion and mobility follows directly from the fluctuation-dissipation theorem.

Passive microrheology measures the motion of the probe due to thermal fluctuations (i.e., the self-diffusvity), and then relates this to an effective viscosity $\eta_{\text {eff }}$ of the medium through an assumed Stokes-Einstein-Sutherland relation

$$
D_{\infty}^{s}=\frac{k_{B} T}{6 \pi \eta_{\mathrm{eff}} a_{h}} .
$$

Generalizations of the above to time- or frequencydependent behavior is straightforward. For a discussion of the relationship between the self-diffusivity and the suspension viscosity, see Ref. 36.

In the nonlinear active microrheology regime, the disturbance to the microstructure is no longer linear in the forcing, but we can still choose to interpret the slowed probe motion as due to an effective "viscosity" of the background suspension. From simple Stokes drag $\boldsymbol{F}^{\mathrm{ext}}=6 \pi \eta_{\mathrm{eff}} a_{h}\langle\boldsymbol{U}\rangle$, we define the effective microviscosity as

$$
\frac{\eta_{\mathrm{eff}}}{\eta}=\left[1-\frac{n k_{B} T}{F_{z}^{\mathrm{ext}}} \oint n_{z} g d S\right]^{-1}
$$

where we have taken the direction of the external force to lie along the $z$-axis. In the case of fixed velocity rather than force, the analog of Eq. (5) becomes

$$
\frac{\eta_{\mathrm{eff}}}{\eta}=1+\frac{n k_{B} T}{6 \pi \eta a_{h} U_{z}^{\mathrm{ext}}} \oint n_{z} g d S .
$$

From (9), it is natural to define the viscosity increment $\Delta \eta$ $=\eta_{\text {eff }}-\eta$ for fixed velocity,

$$
\frac{\Delta \eta_{\mathrm{eff}}}{\eta}=\frac{n k_{B} T}{6 \pi \eta a_{h} U_{z}^{\mathrm{ext}}} \oint n_{z} g d S,
$$

and by analogy for fixed force we have

$$
\frac{\Delta \eta_{\mathrm{eff}}}{\eta}=\frac{n k_{B} T}{F_{z}^{\mathrm{ext}}} \oint n_{z} g d S,
$$

where the latter is strictly true only in the dilute limit when the denominator in (8) can be expanded to leading order in the concentration of bath particles $n$.

It is interesting to compare this microrheological expression for the viscosity to that for macrorheology. The same excluded annulus model in the absence of hydrodynamic interactions gives the following expression for the contribution of the particles to the bulk stress: ${ }^{29}$ 


$$
\left\langle\boldsymbol{\Sigma}_{p}\right\rangle=-n k_{B} T[\boldsymbol{I}+n b \oint \boldsymbol{n n} g(\boldsymbol{r}) d S],
$$

where $\boldsymbol{I}$ is the isotropic tensor. At equilibrium, the contact integral in (12) is isotropic and gives the familiar osmotic pressure of a hard-sphere suspension

$$
\frac{\Pi}{n k_{B} T}=-\frac{1}{3} \boldsymbol{I}:\left\langle\boldsymbol{\Sigma}_{p}\right\rangle=1+4 \phi g(2, \phi),
$$

where $\phi=4 \pi b^{3} n / 3$ is the volume fraction based on the radius $b$ of the bath particles, and only the contact value of the pair-distribution function is important. (For macrorheology there is no probe particle, and all particles are of the same size $b$.) The suspension viscosity is defined as the ratio of the shear stress to shear rate and thus a viscosity increment for macrorheology, analogous to (11), is

$$
\frac{\Delta \eta^{\text {macro }}}{\eta}=-\frac{n^{2} k_{B} T b}{\eta \dot{\gamma}_{x y}} \oint n_{x} n_{y} g(\boldsymbol{r}) d S,
$$

where $\dot{\gamma}_{x y}$ is the imposed shear rate. Here we have subtracted the high-frequency dynamic viscosity, which includes the Einstein correction $(5 / 2) \phi$, but in the absence of hydrodynamic interactions is simply the solvent viscosity $(\eta)$. Note that the macrorheological viscosity increment is for a fixed shear rate, which corresponds to a fixed velocity in microrheology; a fixed stress in macrorheology corresponds to a fixed force in microrheology and would give an expression analogous to (8) for the effective viscosity. Although the fixed stress and fixed shear rate measurements in macrorheology give the same results (apart from any shear banding, etc., i.e., for homogeneously deformed materials), the two situations differ in microrheology as we discuss below.

Apart from the obvious additional factor of $n$ (or $\phi$ ) in the macroviscosity, Eqs. (10) and (14) for the micro- and macroviscosity increments are very similar in structure, involving an appropriate weighting of the pair-distribution function at contact. This similarity is also present when hydrodynamic interactions are included. Although similar, it should be noted that not only are different "moments" of $g$ present in the two expressions, the forcing of the microstructure from equilibrium is also different in the two cases: a dipolar forcing for microrheology and a quadrupolar forcing for macrorheology. Further, the microviscosity also retains an as yet unknown dependence on the size ratio of the probe to the bath particles which is not present in macrorheology. Thus, it is not obvious a priori that the viscosities measured by these two methods should be the same, nor that they should share the same dependence on volume fraction and shear- or pulling-rate. Indeed, a major objective of this work is to explore this connection.

Even without solving for microstructural deformations, it is immediately evident that the viscosity increments measured with the two techniques do scale differently with $\phi$. Physically, this is because the microviscosity arises from collisions between the probe particle and individual bath particles, whereas the macroviscosity increment arises from collisions between bath particles. In the colloidal context, understanding this scaling allows the two measurements to be compared in a meaningful and semi-quantitative fashion. For more general (e.g., unknown) materials, such scalings would not be known a priori, and so the two methods of measurement should not be expected to agree. As a final comment, it should be noted that microrheological experiments can only determine the scalar viscosity (at least for a spherical probe particle, see Sec. X for a discussion). By contrast, macrorheology can, in principle and in practice, determine the full stress tensor, not just the viscosity.

\section{MICROSTRUCTURAL DEFORMATION EQUATION}

To determine the viscosity increment, we need to obtain the pair-distribution function $g(\boldsymbol{r})$ of the bath particles in the presence of a moving probe particle. Although the above expressions for the viscosities are exact for all volume fractions of the bath particles, a closed equation for the pairdistribution function cannot be obtained for all concentrations. To proceed analytically, it is necessary to restrict the analysis to the dilute limit, i.e., to pair-interactions between the probe and bath particles. Below we present a physically motivated derivation of the microstructural evolution equation; a detailed derivation that allows for hydrodynamic interactions and general interaction potentials, can be found in Appendix A.

In a frame fixed on the probe particle, which translates at velocity $\boldsymbol{U}$, each bath particle is advected with velocity $-\boldsymbol{U}$ and moves diffusively. For dilute bath particle concentrations the probability flux has diffusive and advective terms,

$$
\boldsymbol{j}=-D \nabla g-\boldsymbol{U} g,
$$

where $D$ is the relative diffusivity between the bath and probe (with a subtlety to be addressed shortly). Continuity of bath particles requires $\dot{g}+\nabla \cdot \boldsymbol{j}=0$, so that, at steady state, we have the familiar Smoluchowski or advection-diffusion equation

$$
D \nabla^{2} g+\boldsymbol{U} \cdot \nabla g=0 .
$$

Collisions at the probe/bath boundary give rise to a no-flux boundary condition

$$
\boldsymbol{n} \cdot \boldsymbol{j}=D \boldsymbol{n} \cdot \nabla g+\boldsymbol{n} \cdot \boldsymbol{U} g=0 \quad \text { at } r=a+b .
$$

Finally, the microstructure is undisturbed far from the probe, giving

$$
g \sim 1 \quad \text { as } r \rightarrow \infty .
$$

Scaling lengths with the contact distance $[\boldsymbol{r}=(a+b) \hat{\boldsymbol{r}}]$ and velocity with the velocity of an isolated probe $U_{0}$ $=F^{\mathrm{ext}} / 6 \pi \eta a_{h}$, the nondimensional versions of (16)-(18) become

$$
\begin{aligned}
& \nabla^{2} g+P e \partial_{z} g=0, \\
& \left(\partial_{r} g+P e \cos \theta g\right)_{r=1}=0, \\
& g(r \rightarrow \infty) \rightarrow 1,
\end{aligned}
$$

where we have defined a Péclet number 


$$
P e=\frac{U_{0}(a+b)}{D},
$$

and have taken the motion to be along the $z$-axis so that $\hat{\boldsymbol{r}} \cdot \boldsymbol{U}=\cos \theta$. The relative viscosity increment is then given by

$$
\begin{aligned}
\frac{\Delta \eta}{\eta} & =\frac{n k_{B} T(a+b)^{2}}{6 \pi \eta a_{h} U_{0, z}} \oint n_{z} g(r=1 ; P e) d \Omega \\
& =\frac{3}{4 \pi} \frac{D_{a}}{D} \frac{(1+\alpha)^{3}}{P e} \phi \oint n_{z} g(r=1 ; P e) d \Omega,
\end{aligned}
$$

where $\alpha=a / b$ is the ratio of probe to bath radii, $d \Omega$ is the solid angle of integration, $D_{a}$ is the diffisivity of the probe particle, and $D$ is the relative (probe and bath) diffusivity.

Last, we address a subtlety concerning the relative diffusivity $D$. If the velocity of the probe particle is fixed, it experiences no diffusive motion, and relative diffusivity arises from the bath diffusivity alone:

$$
D_{U}=\frac{k_{B} T}{6 \pi \eta b_{h}} \text {. }
$$

By contrast, a probe that is driven through the suspension with a specified force moves both deterministically and diffusively. The relative diffusivity is then given by the sum of the two diffusivities,

$$
D_{F}=D_{a}+D_{b}=\frac{k_{B} T\left(a_{h}+b_{h}\right)}{6 \pi \eta a_{h} b_{h}} .
$$

The difference between $D_{F}$ and $D_{U}$ is negligible when bath particles are much smaller (and more mobile) than the probe particle; in such a case, fixed-force and fixed-velocity measurements give similar results. If the probe is comparable in size to bath particles, however, the distinction becomes appreciable. In fact, constant-force and constant-velocity measurements differ significantly in the small-probe limit. We shall see below that the viscosity increment $\Delta \eta$ in the fixed velocity case exceeds that for fixed force by the amount (1 $+\alpha) / \alpha$, for all $\mathrm{Pe}$. Additional factors arise when hydrodynamic interactions are important (Appendix A), but it appears to be generally true that motion at fixed velocity is more "dissipative" than at fixed force. Such differences have been predicted in falling-ball rheometry of noncolloidal suspensions as well. ${ }^{37}$

\section{MICROSTRUCTURAL DEFORMATION: LIMITING CASES}

Although the Smoluchowski equation (16) can be solved exactly analytically for all $\mathrm{Pe}$, it is instructive to first examine the microstructural deformations that arise in the highand low- $P e$ regimes and the effective viscosity increments that result.

\section{A. Low-Pe limit}

The limit of small $\mathrm{Pe}$ is the linear-response regime in which the microstructure is only slightly perturbed from its equilibrium state. This is the limit that would be probed with passive microrheological techniques. In this case diffusion dominates over advection, and a regular perturbation expan-
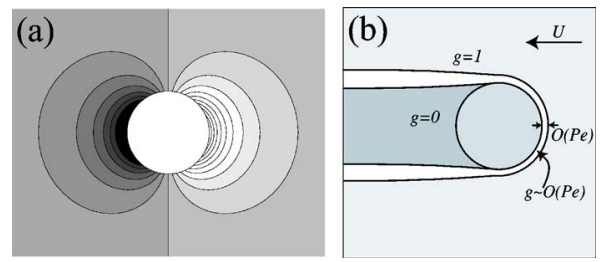

FIG. 2. Low- and high-Pe microstructural deformations. (a) Diffusion dominates at low $P e$, giving a symmetric diffusive dipole and microstructural deformations of order $P e$. (b) A convection-diffusion boundary layer of width $\sim(a+b) P e^{-1}$ forms at high $P e$, within which the deformation has magnitude of order $P e$. The upstream microstructure is unchanged $(g=1)$ outside of the boundary layer, and a wake with no bath particles $(g=0)$ trails the probe.

sion in $P e$ turns the microstructural evolution equation (16) into Laplace's equation with the boundary condition (17) representing simple dipolar forcing

$$
\left.\partial_{r} g\right|_{r=1}=-P e \cos \theta .
$$

The probe particle thus perturbs the microstructure with a simple diffusive dipole,

$$
g=1+P e \frac{\cos \theta}{2 r^{2}}
$$

shown in Fig. 2(a), resulting in a pair-correlation function at contact

$$
g(r=1, \theta)=1+\frac{P e}{2} \cos \theta .
$$

There is an $\mathcal{O}(P e)$ excess of bath particles in front, and an $\mathcal{O}(\mathrm{Pe})$ deficit behind the probe, both of which retard the probe.

\section{B. High-Pe limit}

In the opposite (high $P e$ ) limit, diffusion is important only in a thin boundary layer of thickness $\delta \sim \mathcal{O}\left(P e^{-1}(a\right.$ $+b)$ ) adjacent to the probe, outside of which advection dominates. In this limit, the boundary layer looks locally planar, and gradients along the boundary layer are small compared with those across it. Diffusion balances the perpendicular component of the advection velocity $\left(u_{\perp}=U \cos \theta\right)$, giving an approximate equation

$$
D \frac{\partial^{2} g}{\partial \zeta^{2}}+U \cos \theta \frac{\partial g}{\partial \zeta}=0,
$$

where $\zeta$ is a coordinate perpendicular to the (local) surface. This has the solution

$$
g(\boldsymbol{r}) \sim 1+f(\theta) e^{-U \zeta \cos \theta / D},
$$

where $f$ is a function to be determined. One can integrate across the boundary layer to obtain the "local" surface probability density $\sigma(\theta)$,

$$
\sigma(\theta)=\int_{0}^{\infty} f(\theta) e^{-U \cos \theta \zeta / D} d \zeta=\frac{D f(\theta)}{U \cos \theta} .
$$




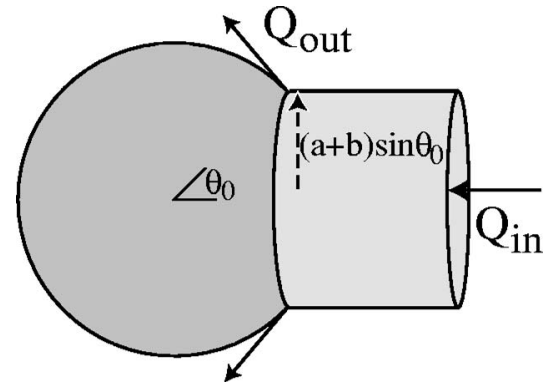

FIG. 3. The microstructure within the high- $P e$ boundary layer can be determined by a flux balance. A total flux $Q_{\text {in }}=g^{\infty} U \pi(a+b)^{2} \sin ^{2} \theta_{0}$ flows through the cylinder into the boundary layer. Transport within the boundary layer, along the contact surface $(r=a+b)$, occurs due to the parallel component of the velocity, $U_{\|}=U \sin \theta$, which sweeps a flux $Q_{\text {out }}=2 \pi(a+b) \sin \theta_{0} U_{\|} \sigma\left(\theta_{0}\right)$ out of the cylinder. Here $\sigma$ is the local microstructure probability density, integrated across the boundary layer. Requiring $Q_{\text {in }}$ to equal $Q_{\text {out }}$ in steady state gives (35).

The function $\sigma\left(\theta_{0}\right)$ at any angle $\theta_{0}$ can be determined using the flux balance shown in Fig. 3. The total flux into the $\theta<\theta_{0}$ portion of the boundary layer is simply given by

$$
Q_{\text {in }}\left(\theta_{0}\right)=\pi\left[(a+b) \sin \theta_{0}\right]^{2} U g^{\infty} .
$$

In steady state, the inward flux $Q_{\text {in }}$ must be balanced by the flux $Q_{\text {out }}$ of particles advected out of the boundary layer. The parallel component of the velocity, $U_{\|}=U \sin \theta_{0}$, carries the probability $\sigma\left(\theta_{0}\right)$, outside of the control volume. The total probabilty, integrated around the perimeter at $\theta_{0}$, is $2 \pi(a$ $+b) \sin \theta_{0} \sigma\left(\theta_{0}\right)$, so that $Q_{\text {out }}$ is given by

$$
Q_{\text {out }}\left(\theta_{0}\right)=2 \pi(a+b) \sigma\left(\theta_{0}\right) U \sin ^{2} \theta_{0}
$$

downstream along the surface. Requiring that $Q_{\text {in }}=Q_{\text {out }}$ at steady state gives

$$
\sigma\left(\theta_{0}\right)=\frac{(a+b) g^{\infty}}{2},
$$

meaning that $\sigma$ is constant on the leading hemisphere, but zero on the trailing hemisphere, as shown in Fig. 2(b).

The upstream perturbed microstructure follows from (30), (31), and (34),

$$
g\left(\boldsymbol{r}, \theta<\frac{\pi}{2}\right) \sim 1+\frac{P e}{2} \cos \theta e^{-U z \cos \theta / D}
$$

giving a pair correlation at contact

$$
g\left(r=1, \theta<\frac{\pi}{2}\right)=1+\frac{P e}{2} \cos \theta .
$$

The pair correlation at contact on the downstream face is zero. Thus there is a large $\mathcal{O}(\mathrm{Pe})$ excess in front of the probe, but only an $\mathcal{O}(1)$ deficit behind it, since the bath particle concentration cannot drop below zero.

\section{Relative viscosity increments in both limits}

Finally, we use (28) and (36) in (23) to calculate the relative viscosity increment in the two limits, giving

$$
\frac{\Delta \eta(P e \ll 1)}{\eta}=2 \frac{\Delta \eta(P e \gg 1)}{\eta}=\phi \frac{(1+\alpha)^{3}}{2} \frac{D_{a}}{D} .
$$

Notably, the high- and low-Pe limits differ only by a factor of 2, despite the substantial qualitative differences in their microstructures. This reflects the singular nature of $P e \rightarrow \infty$ : the advection-diffusion boundary layer shrinks like $P e^{-1}$, yet the concentration within the boundary layer grows like $P e$, giving a finite contribution even as $P e \rightarrow \infty$. The decrease in the viscosity with increasing shear- or pulling-rate ("shear thinning") is typical of colloidal suspensions. Since $g(1)$ $\sim \mathcal{O}(P e)$ for all $P e$, and since the dimensionless viscosity increment has a $P e^{-1}$ in front of the microstructural integral [Eq. (23)], the correction is always $\mathcal{O}(1)$.

A clear physical picture of shear thinning emerges from this example. On a physical level, shear thinning occurs because at low $P e$, the bath particle is both pushed from the front and sucked from behind; by contrast, the high- $P e$ microstructural perturbation is $\mathcal{O}(\mathrm{Pe})$ only in the leading-edge boundary layer, and only $\mathcal{O}(1)$ behind, and thus the probe is only "pushed" from the front-a factor of 2 smaller that at low $P e$. It may seem strange that the effective viscosity is larger at small rather than high $P e$, where there is a large buildup of bath particles in front of the probe which would seem to offer strong resistance to motion. However, in the high- $\mathrm{Pe}$ limit, the entropic reactive force of the bath particles is $\mathcal{O}\left(k_{B} T /(a+b)\right)$, which is much smaller than the external driving force, $\boldsymbol{F}^{e x t}$ - their ratio being $P e^{-1}$. Therefore, the reactive force would go to zero in the limit of high $P e$, were it not for the boundary layer at contact with $\mathcal{O}\left(P e^{-1}\right)$ thickness and $\mathcal{O}(P e)$ amplitude, which amplifies the entropic reactive force to be $\mathcal{O}\left(P e k_{B} T /(a+b)\right)$ - the same size as the driving force.

In what follows, we highlight two important features of active microrheology: a dependence on probe size, and the difference between viscosity increments measured in the two "ideal" modes of active microrheology (fixed velocity and fixed force). Using (24) and (25)), we note that

$$
\frac{D_{a}}{D_{F}}=(1+\alpha)^{-1}
$$

for the constant-force probe, and

$$
\frac{D_{a}}{D_{U}}=\alpha^{-1}
$$

for the constant-velocity probe. A low-Pe fixed-velocity probe would measure an effective viscosity increment

$$
\frac{\Delta \eta^{U}(P e \ll 1)}{\eta}=\phi \frac{(1+\alpha)^{3}}{2} \frac{b_{h}}{a_{h}}=\phi \frac{(1+\alpha)^{3}}{2 \alpha},
$$

whereas the low-Pe fixed-force probe would measure

$$
\frac{\Delta \eta^{F}(P e \ll 1)}{\eta}=\phi \frac{(1+\alpha)^{2}}{2} .
$$

The ratio of the viscosity increments at fixed velocity and fixed force is 


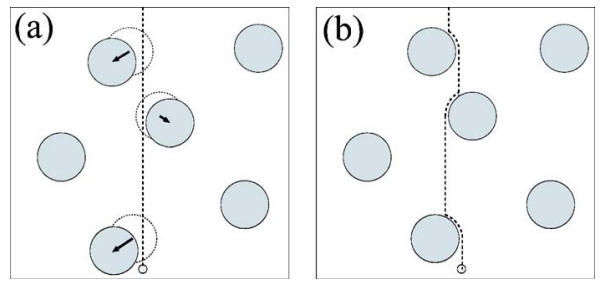

FIG. 4. When the probe is smaller than the bath particles $(a<b$, or $\alpha \ll 1)$, the difference between fixed-velocity and fixed-force modes becomes apparent. (a) If the probe is constrained to move at fixed velocity (the "bulldozer" limit), it must push all bath particles out of its way, increasing the force on the probe substantially during collisions. (b) A probe moving under a fixed applied force (the "Pachinko" limit) can move laterally to pass the large and immobile bath particles. Since the probe velocity remains of order $U$ throughout the collisions, the change to the velocity is not as dramatic.

$$
\frac{\Delta \eta^{U}}{\Delta \eta^{F}}=\frac{1+\alpha}{\alpha},
$$

and simply represents the ratio of the characteristic time scales $\tau_{U} / \tau_{F}=D_{F} / D_{U}$. The high-Pe limits are simply $1 / 2$ of (40) and (41), while the ratio (42) applies for all $P e$.

We now examine the influence of particle size ratio on the viscosity increment. The two measurements are the same in the large-probe limit $\alpha \rightarrow \infty$, giving $\Delta \eta \sim \alpha^{2}$. This can be understood in the high- $P e$ (deterministic) limit physically as follows: A large probe moves a distance $L$ in a time $\tau_{L}$ $\sim L / U$, during which it collides with $N \sim \alpha^{2} L \phi / b$ bath particles. Each collision endures for a time $\tau_{c} \sim a / U$, during which it contributes an additional retarding force $\Delta F$ $\sim F_{0} / \alpha$. The time-averaged force is simply $\langle\Delta F\rangle$ $\sim N \tau_{c} \Delta F / \tau_{L}$, giving $\langle\Delta F\rangle / F_{0} \sim \alpha^{2} \phi$. In this limit, the probe acts like a large "strainer" - a limit which may be difficult to achieve in practice due to the finite extent of intercolloidal forces.

When $\alpha \sim \mathcal{O}(1)$ or smaller, however, the two techniques give rather different results. In this limit, the force required to push bath particles (relative to the Stokes force on the probe) is large. As shown in Fig. 4(b), a fixed-force probe can make its way around bath particles without displacing them, like a ball falling through a Pachinko machine, and the velocity remains of $\mathcal{O}(U)$ throughout the collision. A fixedvelocity probe, on the other hand, must push the large bath particles out of its way to maintain its constant velocity [Fig. 4(a) _ - like a bulldozer - and requires a force that is a factor of $\mathcal{O}(b / a)$ larger than the Stokes force on a probe.

The bulldozer and Pachinko limits can be understood in the high- $P e$, low- $\alpha$ limit from a simple physical picture. The bulldozer limit is simplest: in traversing a distance $L$, the probe experiences $N \sim(1+\alpha)^{2} L \phi / b$ collisions, each of which lasts a time $\tau_{c} \sim(a+b) / U$ and contributes a force $F_{0} / \alpha$. The average force experienced by the probe is then increased by an amount $\langle\Delta F\rangle / F_{0} \sim(1+\alpha)^{3} \phi / \alpha$, as in (40).

The fixed-force (Pachinko) limit is similar, but more subtle: consider $L \sim b / \phi$ to be the distance the probe must travel before colliding with a bath particle, so that on average the probe collides once per $L$, with a collision that lasts $\tau_{c}$ $\sim b / U$. During collisions, the probe velocity is slowed by a factor of order $U$, giving a relative velocity increment

$$
\langle\Delta U\rangle \sim\left(\frac{U \tau_{c}}{L / U}\right) \sim \phi,
$$

that remains finite in the limit $\alpha \rightarrow 0$, as in (41).

Hydrodynamic interactions modify the above dependence on size ratio $\alpha$. Previous studies have been performed in the non-Brownian limit $(P e=\infty)$ in the context of falling ball rheometry. ${ }^{37}$ Incorporating hydrodynamic interactions yields the Einstein result $\Delta \eta / \eta \rightarrow 5 \phi / 2$ in the large-probe limit $(\alpha \rightarrow \infty)$, as expected. A divergent viscosity is obtained in the non-Brownian, small probe limit $(\alpha \rightarrow 0)$, for both constant force and constant velocity modes (in contrast with our results, where the divergence only occurs for the constant velocity mode). Physically, the $P e=\infty, \alpha \ll 1$ divergence arises because hydrodynamic interactions decay on the scale of the (larger) bath particles, so that "collisions" are longlasting. Lubrication interactions dramatically slow the probe when it is closest to bath particles (or increase the force necessary to maintain a constant velocity), further enhancing the average retarding effect. By contrast, the present theory (which neglects hydrodynamic interactions) yields a probe velocity that remains $\mathcal{O}(U)$ even at contact and results in a finite viscosity for constant-force measurements in the $\lambda$ $\gg 1$ limit. Which picture is more relevant to experiments depends on the physically realizable values of $\lambda$.

\section{FLUCTUATIONS}

The above calculations used the probability distribution function for the steady-state microstructure to determine an average quantity - the viscosity increment $\Delta \eta$. However, the $N$-particle distribution function $P_{N}$ (Appendix A) allows any statistical quantity to be calculated. Here, we examine the fluctuations about the mean.

The velocity increment for a particular microstructural realization is given by

$$
\frac{\Delta \boldsymbol{U}}{U}=-\frac{k_{B} T}{6 \pi \eta a_{h} U} \nabla \ln P_{N},
$$

for a constant-force probe. As calculated above, an average (weighted by $P_{N}$ ) gives the ensemble-averaged relative velocity increment. The difference between the velocity increment due to a particular microstructure and the average increment is given by

$$
\Delta \boldsymbol{U}^{\prime}=\Delta \boldsymbol{U}-\langle\Delta \boldsymbol{U}\rangle,
$$

and fluctuations follow from the mean-square velocity variation,

$$
\left\langle\Delta \boldsymbol{U}^{\prime 2}\right\rangle=\left\langle\Delta \boldsymbol{U}^{2}\right\rangle-\langle\Delta \boldsymbol{U}\rangle^{2},
$$

giving

$$
\begin{aligned}
\frac{\left\langle\Delta \boldsymbol{U}^{\prime 2}\right\rangle}{U^{2}}= & \frac{\left\langle\Delta \boldsymbol{F}^{\prime 2}\right\rangle}{F_{0}^{2}} \equiv \Delta=\left(\frac{D_{a}}{U}\right)^{2} n \int \nabla g \nabla g(1 / g) d V \\
& +\mathcal{O}\left(\phi^{2}\right) .
\end{aligned}
$$

Note that $\langle\Delta \boldsymbol{U}\rangle^{2}$ is omitted from the right-hand side because it is of order $\phi^{2}$. Nondimensionalizing, we obtain 


$$
\Delta=\frac{3}{4 \pi} \phi \frac{(1+\alpha)^{3}}{P e^{2}}\left(\frac{D_{a}}{D}\right)^{2} \iint_{1}^{\infty} \frac{\nabla g \nabla g}{g} r^{2} d r d \Omega
$$

Before explicitly calculating the fluctuations, we discuss briefly their scaling. In the small-Pe limit, $g \sim \mathcal{O}(1)$ and $\nabla g \sim \mathcal{O}(P e)$, giving an $\mathcal{O}\left(P e^{2}\right)$ integral. In the large- $P e$ limit, $g$ is largest in the thin boundary layer of volume $\mathcal{O}\left(P e^{-1}\right)$ around the leading edge of the probe, where $g \sim \mathcal{O}(P e)$ and $\nabla g \sim \mathcal{O}\left(P e^{2}\right){ }^{38}$ Thus the integral in (48) is $\mathcal{O}\left(P e^{2}\right)$ in both limits, and fluctuations are of order

$$
\Delta \sim \phi(1+\alpha)^{3}\left(\frac{D_{a}}{D}\right)^{2} .
$$

Using (37) and (49), we see that the relative fluctuations (normalized by the increments) have the scaling

$$
\frac{\Delta^{1 / 2}}{\Delta U / U} \sim \frac{1}{\phi^{1 / 2}(1+\alpha)^{3 / 2}} .
$$

Relative fluctuations diverge as $\phi \rightarrow 0$ when collisions are infrequent, and vanish in the large-probe limit due to the large number of colliding bath particles. In the small-probe limit $(\alpha \rightarrow 0)$, on the other hand, the fixed-velocity fluctuations diverge in the same way $\left(\sim \alpha^{-1}\right)$ that the increment itself diverges. This reflects the physics of this ("bulldozer") limit: the force on the probe alternates between the relatively small Stokes force between collisions and the large forces required to push bath particles out of the way. Here the mean is dominated by the fluctuations, and the two scale in the same way.

It is fairly straightforward to evaluate (48) using the microstructures derived for low and high $P e$, Eqs. (27) and (35). The fluctuations are given by

$$
\begin{aligned}
& \Delta_{i j}(P e \ll 1)=\frac{(1+\alpha)^{3}}{20}\left(\frac{D_{a}}{D}\right)^{2} \phi\left(3 \delta_{i j}+\hat{U}_{i} \hat{U}_{j}\right), \\
& \Delta_{i j}(P e \gg 1)=\frac{(1+\alpha)^{3}}{20}\left(\frac{D_{a}}{D}\right)^{2} \phi\left(2 \delta_{i j}+\hat{U}_{i} \hat{U}_{j}\right),
\end{aligned}
$$

where $\hat{\boldsymbol{U}}$ is a unit vector in the direction of $\boldsymbol{U}$. The ratio of parallel to perpendicular fluctuations is given by

$$
\begin{aligned}
& \frac{\Delta_{\|}}{\Delta_{\perp}}(P e \ll 1)=\frac{4}{3}, \\
& \frac{\Delta_{\|}}{\Delta_{\perp}}(P e \gg 1)=\frac{3}{2} .
\end{aligned}
$$

\section{SOLUTION FOR ARBITRARY Pe}

Having explored the two limiting cases, we now proceed to treat the case of general $P e$, the details of which can be found in Appendix B. Microstructural deformations for three intermediate values of $P e$, obtained using this solution, are shown in Fig. 5. The viscosity increment,
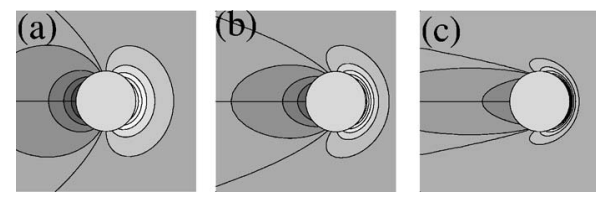

FIG. 5. Microstructural perturbations at intermediate $P e$, found using the general solution (B1). (a) Asymmetry is notable at $P e=0.15$, (b) increases for $P e=0.5$, and (c) a wake becomes evident for $P e=1.5$.

$$
\frac{\Delta \eta}{\eta}=\frac{(1+\alpha)^{3}}{2} \frac{D_{a}}{D} \phi V(P e)
$$

can be calculated for intermediate $P e$, with the dimensionless viscosity increment function $V(P e)$ given to $\mathcal{O}\left(P e^{4}\right)$ by

$$
V(P e)=\left[1-\frac{2}{15} P e^{2}+\frac{1}{8} P e^{3}-\frac{128}{1575} P e^{4}\right]+\mathcal{O}\left(P e^{5}\right) .
$$

Equation (56) agrees with Eq. (37) as expected in the low- $P e$ limit, but diverges at high $P e$, reflecting the singular nature of the advection-diffusion problem. While the exact solution in Appendix B is valid for arbitrary Pe, the singular nature of the problem renders its implementation in the high$P e$ limit less practical. From (B13) a 19-term expansion of $V(P e)$ was obtained and Padé approximants used to extrapolate to the high- $P e$ limit. A (9-9) Pade approximant, plotted in Fig. 6, reveals $V(P e \rightarrow \infty)=0.5019$, quite close to the expected value of $1 / 2$. Force-thinning behavior is clearly evident. We expect Fig. 6 will represent a sort of "universal curve" for active microrheology of colloidal suspensions when $\alpha \sim \mathcal{O}(1)$, after accounting for probe/bath sizes and volume fractions as in (55), and higher volume fractions treated as described in Sec. IX.

The general solution can likewise be used to calculate the fluctuations for arbitrary $P e$, giving, to leading order in $P e$,

$$
\begin{aligned}
& \Delta_{\|} \sim \frac{(1+\alpha)^{3}}{5}\left(\frac{D_{a}}{D}\right)^{2} \phi U^{2}\left(1-\frac{353}{2940} P e^{2}+\cdots\right), \\
& \Delta_{\perp} \sim \frac{3(1+\alpha)^{3}}{20}\left(\frac{D_{a}}{D}\right)^{2} \phi U^{2}\left(1-\frac{209}{1470} P e^{2}+\cdots\right) .
\end{aligned}
$$

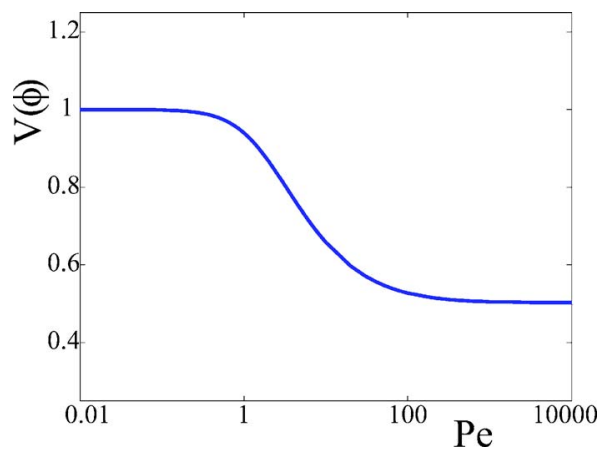

FIG. 6. The 9-9 Pade approximant to the dimensionless viscosity increment function $V(P e)$. A clear force-thinning behavior is evident, with a high$\mathrm{Pe} V(\mathrm{Pe})$ asymptote of $1 / 2$. We expect that the Brownian viscosity contribution for a wide range of active microrheology experiments will collapse onto this "universal curve," once probe size and bath volume fraction are properly scaled out. Note also that the analogous (macrorheological) shear viscosity shear-thins in a similar, but not identical, fashion. 
Several features in the complete expression for the viscosity increment, Eq. (55), bear emphasis. As discussed above, the factor $\left(D_{a} / D\right)$ depends on how the probe is pulled (constant force or constant velocity), most dramatically for probes that are as small as or smaller than bath particles. It will be important to keep this distinction in mind when interpreting experiments. Second, the Péclet number itself [Eq. (22)] depends on the relative diffusion constant, and thus on both particle size and driving mode [Eqs. (24) and (25)]. From the constant-velocity and constant-force Péclet numbers,

$$
P e_{U}=\frac{U(a+b)}{D_{b}}, \quad P e_{F}=\frac{U a}{D_{b}},
$$

one sees again that the small-probe limit is special: pulling the probe at the same velocity, but in different modes, yields different $P e$ and thus different microstructural deformations. In general, $P e_{F}$ is smaller than $P e_{U}$, reflecting the additional probe diffusivity in the constant-force mode.

Equation (59) raises another subtlety: in computing the constant-force Péclet number $P e_{F}$, what velocity $U$ should be used? Since the difference between the probe velocity in suspension and its Stokes velocity is $\mathcal{O}(\phi)$, no significant difference occurs in the dilute limit $\phi \ll 1$, and $P e_{F}=F(a$ $+b) / k_{B} T$. However, at higher volume fractions the situation may be different. This is discussed in detail in Sec. IX, where a proposal for scaling-up to higher $\phi$ is given.

\section{MIXED MODE}

Thus far, we have considered "idealized" active microrheology in which the probe particle is driven at either fixed velocity, or with a fixed force. In real experiments, however, neither mode may occur. For example, a probe particle held in place by optical or magnetic gradient forces while the bath itself is translated might seem to be a fixedvelocity probe. In fact, however, such probes are held in a (roughly) harmonic potential well, and can make excursions from the center of the trap. Whether a probe behaves like fixed-velocity or fixed-force depends on the "stiffness" of the trapping force. This can be understood most clearly in the small-probe, non-Brownian limit, where the distinction between experimental modes is strongest.

Consider a small probe $(\alpha \ll 1)$ held in a harmonic potential centered at $\boldsymbol{x}=0$, against a bath translating with velocity $\boldsymbol{U}_{\text {bath }}$, and subject to a harmonic restoring force $\boldsymbol{F}=-k \boldsymbol{x}$. A translating probe particle experiences Stokes drag $F_{s}$ $=6 \pi \eta a_{h} U_{\text {bath }}$, and trails the center of the trap by a distance $\Delta x=F_{s} / k$. When the probe collides with a (large) bath particle, its behavior depends on the strength of the trapping potential. If the spring is very strong, the probe is held in place and can "bulldoze" the bath particle out of the way. A weak spring, on the other hand, allows the probe to temporarily leave the trap center to make an excursion around the bath particle.

In the Pachinko (constant-force) limit, the bath particle remains fixed during the collision, and the probe moves a distance of order $\mathcal{O}(a+b)$ from its steady-state position, which changes the force on the probe by an amount of order $\Delta F \sim k(a+b)$. The constant-force limit thus occurs when $\Delta F \ll F_{s}$, or

$$
U_{\text {bath }} \gg \frac{k(a+b)}{6 \pi \eta a_{h}} \equiv U_{F}^{c} .
$$

The opposite (bulldozer) limit occurs when the spring is strong enough to hold the probe in place as it pushes the bath particle aside. In that case, both particles are forced to move with velocity $\sim U_{\text {bath }}$, which requires an additional force $\Delta F \sim 6 \pi \eta b_{h} U_{\text {bath }}$ acting back on the probe. If the resulting displacement of the probe, $\Delta x \sim 6 \pi \eta b_{h} U_{\text {bath }} / k$, is small compared to the collision radius $(a+b)$, then the spring force is sufficient to hold the probe in place while it bulldozes the bath particle. Thus the fixed-velocity limit occurs when

$$
U_{\text {bath }} \ll \frac{k(a+b)}{6 \pi \eta b_{h}}=\alpha U_{F}^{c} .
$$

To summarize, in the non-Brownian $(P e \gg 1)$, smallprobe $(\alpha \ll 1)$ limit, translating the trap slowly $\left(U<\alpha U_{F}^{c}\right)$ corresponds to fixed-velocity operation, quickly $\left(U>U_{F}^{c}\right)$ corresponds to fixed-force operation, and with intermediate speeds $\alpha<U / U_{F}^{c}<1$ corresponds to a mixed-mode of operation. All three may occur in real active microrheology experiments as pulling speeds increase.

To see how these limits arise, consider the mixed-mode problem for the joint probability $P$ of finding the probe particle at location $\boldsymbol{r}_{1}$ relative to the trap and a bath particle at $\boldsymbol{r}_{2}$ relative to the probe. The detailed derivation, including hydrodynamic interactions, is given in Appendix A.

The probe and the bath particle fluxes, viewed from the frame of the moving trap, are given by

$$
\begin{aligned}
& \boldsymbol{j}_{1}=-k_{B} T M_{a} \nabla_{1} P-M_{a} k r_{1} P-U_{\text {bath }} P, \\
& j_{2}=-k_{B} T M_{b} \nabla_{2} P-U_{\text {bath }} P,
\end{aligned}
$$

and probability conservation yields

$$
\begin{aligned}
& k_{B} T M_{a} \nabla_{1}^{2} P+k_{B} T M_{b} \nabla^{2} P+\boldsymbol{U}_{\text {bath }} \cdot\left(\nabla_{2} P+\nabla_{1} P\right) \\
& \quad+M_{a} k \nabla \cdot\left(\boldsymbol{r}_{1} P\right)=0 .
\end{aligned}
$$

The no-flux boundary condition is quite complicated in this frame, but is simpler in the probe frame; in that case, however, Eq. (64) becomes more complicated (see Appendix A for details).

Scaling mobilities by the probe mobility $M_{a}$ and lengths by $(a+b)$ gives

$$
\nabla_{1}^{2} P+\alpha \nabla_{2}^{2} P+P e \hat{\mathbf{z}} \cdot\left(\nabla_{2} P+\nabla_{1} P\right)+K \nabla_{1} \cdot\left(\boldsymbol{r}_{1} P\right)=0,
$$

where $P e$ is based on probe diffusivity and where we have introduced a nondimensional spring constant

$$
K=\frac{\alpha k(a+b)^{2}}{k_{B} T} .
$$

The above physical picture, which invoked the small-probe limit $\alpha \ll 1$, can be understood from Eq. (65). Because $\alpha$ $\ll 1$, we neglect the second term but retain the first (diffu- 
sive) term, which can balance either the third (convective) or fourth (spring). Whether the convective or spring flux is dominant is determined by the ratio

$$
\frac{K}{P e}=\frac{k(a+b)}{6 \pi \eta b_{h} U_{\text {bath }}} .
$$

The condition $K \gg P e$ is equivalent to Eq. (61), and corresponds to the constant velocity mode: the spring is strong enough to hold the probe in place. The opposite (fixed-force) limit, Eq. (60), corresponds to $K \ll \alpha P e$, for which the origin [in Eq. (65)] of the additional $\alpha$ is not immediately clear. In general, a more detailed analsis of Eq. (65) [or Eqs. (A31) and (A32)] will be required to determine the fixed-force and fixed-velocity conditions for general $\alpha$. The problem seems to be particularly rich-K, $\mathrm{Pe}, \alpha$-with a number of possible interesting limits.

\section{RHEOLOGY}

\section{A. Comparison to macrorheology}

A major issue in the use of microrheology as a predictive and/or characterization tool is to what extent the microviscosity and the macroviscosity are the same. The expressions (10) and (14) for the viscosity increments clearly suggest a similarity, as does the force-thinning of the microviscosity; colloidal suspensions without hydrodynamic interactions also shear thin ${ }^{31}$ and have a similar $\mathcal{O}(1 / P e)$ boundary layer at particle-particle contact at high Peclet number. ${ }^{29}$ But exactly how good is the connection?

In the linear-response regime of passive microrheology, the measurement of the mean-square displacement corresponds to the transition from the short- to the long-time selfdiffusivity - high frequency gives short time and low frequency gives long time (see Appendix A). This problem has been studied extensively in the colloids literature for a diffusing particle of the same size as the "background" particles $(\alpha=1)$, which form a viscoelastic medium (e.g., see Ref. 36). The frequency-dependent viscosity of such a medium has also been studied by macroscopic rheology 39 and thus a direct comparison between micro and macro is possible. Since the transition from one limit to another reflects the underlying microstructural behavior, the proper comparison is between the scaled differences: (i) micro: $\left[1 / D^{s}(\omega)\right.$ $\left.-1 / D_{0}^{s}\right] /\left(1 / D_{\infty}^{s}-1 / D_{0}^{s}\right)$ and (ii) macro: $\left[\eta(\omega)-\eta_{\infty}^{\prime}\right] /\left(\eta_{0}\right.$ $\left.-\eta_{\infty}^{\prime}\right)$. Here $D_{0}^{s}$ is the short-time self-diffusivity, $\eta_{\infty}^{\prime}$ is the high-frequency dynamic viscosity and $\eta_{0}$ is the steady zeroshear-rate viscosity. This comparison was addressed in Ref. 36 where it was shown that, apart from the obvious different volume fraction scalings in the dilute limit (micro scales with $\phi$, while macro scales with $\phi^{2}$ ), the micro- and macroviscosities agree very well for all frequencies and volume fractions. It is also the case that $D_{\infty}^{s} \times \eta_{0}$ and $D_{0}^{s} \times \eta_{\infty}^{\prime}$ are experimentally found to be approximately constant for all $\phi$. Thus, in the linear-response regime the micro-macro connection is semi-quantitatively well founded. [It is also interesting to note that the linear response viscosities (micro and macro) frequency-thin in a manner similar to shear thinning and possess a high-frequency boundary-layer at particle-particle contact analogous to the that at high $P e^{36,39,40}$ No analogous

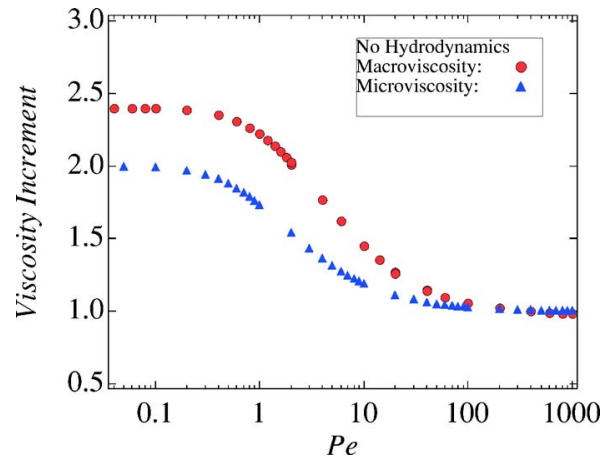

FIG. 7. The macroviscosity increment, $\left(\eta^{\text {macro }} / \eta-5 \phi / 2-1\right) / \phi^{2}$, of Bergenholtz et al. (Ref. 30) in absence of hydrodynamic interactions $(\lambda \rightarrow \infty)(\mathbf{O})$ is plotted vs the Péclet number along with the microviscosity increment, $\left(\eta^{\text {micro }} / \eta-1\right) / \phi$, determined in this work $(\triangle)$. We have taken $\alpha=1$ for comparison. For macrorheology $P e=\dot{\gamma} a^{2} / D_{a}$, where $\dot{\gamma}$ is the shear rate, and for microrheology, $P e=U a / D_{a}$. While the agreement shown here is nearly quantitative, we emphasize that the similarity in the shear thinning behavior is the most relevant comparison to make, as detailed amplitudes depend on $\alpha$, perhaps $\lambda$ for large $\alpha$, and so on. Furthermore, we have scaled the relative viscosity increments appropriately with $\phi$, which is only possible because such scalings are known for collodal suspensions. For more general (and unknown) complex fluids, such scalings would not be known a priori.

shear- (or frequency-) thickening appears, however, when the departure from equilibrium is small, regardless of frequency, i.e., small Péclet number.]

In the nonlinear regime of active microrheology, we can make the first definitive connection between micro and macro by comparing with the work of Bergenholtz et al., ${ }^{30}$ who considered the analogous macrorheology problem-a dilute, monodisperse suspension of spheres interacting with the excluded annulus model. They solved the appropriate pair Smoluchowski equation and determined the effective macroviscosity increment (14). Their macroviscosity increments, $\left(\eta^{\text {macro }} / \eta-5 \phi / 2-1\right) / \phi^{2}$, in the absence of hydrodynamic interactions $(\lambda \rightarrow \infty)$ are shown in Fig. 7 along with our results for the microviscosity increments, $\left(\eta^{\text {micro }} / \eta\right.$ -1)/ $\phi$, as functions of $P e: P e=\dot{\gamma} a^{2} / D_{a}$ for macrorheology, where $\dot{\gamma}$ is the shear rate, and $P e=U a / D_{a}$ for microrheology. Note that we have taken $\alpha=1$, or $a=b$, in making this comparison.

As seen from the comparison, both viscosities "shear" thin and reach the same high Péclet number asymptote, which is a manifestation of the $\mathcal{O}(1 / P e)$ boundary layers present in both cases. The low Péclet asymptotes are different, however, being $12 / 5$ for the macroviscosity and 2 for the microviscosity, reflecting the difference between the dipolar and quadrupolar forcings. Apart from these slight numerical differences, the microviscosities are in very good accord with the macroviscosities, so long as the appropriate scaling with $\phi$ is performed and $\alpha \sim \mathcal{O}(1)$. As $\alpha$ increases, $\eta^{\text {micro }}$ would increase as well, as the probe acts like a "strainer" so long as $\lambda \rightarrow \infty$. In the large-probe $(\alpha \gg 1)$ limit, the $\lambda=\infty$ approximation may no longer be appropriate.

In the absence of hydrodynamic interactions, the viscosity (micro or macro) shear thins; hydrodynamic lubrication interactions are required for shear thickening. As shown by Bergenholtz et al $^{30}$ for the excluded annulus model the macroviscosity shear thins for all $\lambda \geqslant 1.1$; only for $\lambda<1.1$ is 
shear thickening seen. The recent work of Khair and Brady ${ }^{34}$ shows the same to be true for the microviscosity.

Thus, it seems that, at least for dilute colloidal dispersions, the macro- and microviscosities are comparable for all Péclet numbers and display both shear or force thinning, as well as shear or force thickening when hydrodynamic lubrication forces become important $(\lambda<1.1)$. Although the micro- and macroviscosities are comparable, it should be noted that in microrheology only the scalar viscosity (at least for a single spherical probe particle) can be determined. In contrast, a macrorheology experiment or theory can determine the full stress tensor, including normal stress differences and an isotropic pressure. This is an important distinction that must be borne in mind when assessing the strengths and weaknesses of the two approaches.

The comparison shown in Fig. 7 is between the microviscosity at fixed force and the macroviscosity at fixed shear rate. Properly, one should compare fixed velocity with fixed shear rate (or fixed force with fixed stress). However, at the pair level, i.e., to leading order in $\phi$, the macroviscosity at fixed stress is the same as at fixed shear rate and so the comparison in Fig. 7 is appropriate. Even though this mixed comparison is legitimate, the microviscosties for fixed force and fixed velocity are not the same at leading order in $\phi$; from Eq. (55) we see that the microviscosity at fixed velocity (for $a=b$ ) is a factor of 2 larger than that at fixed force for all $P e$. Also, the Péclet number for fixed velocity is twice as large as that at fixed force. Together this would shift the microviscosities in Fig. 7 up and to the right by a factor of 2 . The important comparison is the form of the shear thinning behavior, not necessarily the detailed amplitude.

That the microviscosities at fixed force and fixed velocity are different naturally raises the question as to whether the marcoviscosities for fixed stress and fixed shear rate may also differ. It is easy to show that in the linear-response regime fixed stress and fixed shear rate give the identical macroviscosities. In the nonlinear regime, however, one cannot prove that the two are the same, although it would seem most likely that they are. (We are considering here homogeneously deformed materials and exclude from discussion nonhomogeneous deformations such as shear banding, etc., which could differ in the two situations.) In the thermodynamic limit of large systems the fluctuations in shear rate that are present for experiments at constant stress are expected to decay as $1 / \sqrt{N}$ and thus vanish, resulting in the same viscosity for the two systems. If the fluctuations did not decay with system size, as might happen near a critical point (a dispersion near a point of yielding, e.g.) then the two measurements may give different macroviscosities. By contrast, in microrheology the constant force or velocity is applied to a single probe particle, and the differing fluctuations in the two situations are not small on the scale of the probe, therefore giving rise to measurable differences in response.

\section{B. Scale-up to higher concentrations}

Although we have considered the simplest, ideal, case of a dilute suspension of hard spheres without hydrodynamic interactions, previous work on the macrorheology of colloi- dal dispersions ${ }^{29,31,41}$ has shown that the results of such a model can be scaled up to accurately predict the behavior of concentrated dispersions both with and without hydrodynamic interactions. Here, we follow the same reasoning and offer suggestions as to how the present results may be extended to higher concentrations and to include hydrodynamic interactions.

There are several issues that need to be appreciated before attempting to scale up dilute results to higher concentrations. Both the magnitude of the viscosity and the Péclet number need to be scaled. Furthermore, it is the viscosity increments that one should try to scale up and compare between micro and macro, as these reflect the microstructural deformations that produce the rheological behavior. For macrorheology, the viscosity increment is $\Delta \eta^{\text {macro }}=\eta^{\text {macro }}(\mathrm{Pe})$ $-\eta_{\infty}^{\prime}$, where $\eta_{\infty}^{\prime}$ is the high-frequency dynamic viscosity, which is a purely hydrodynamic quantity; in the absence of hydrodynamics $(\lambda \rightarrow \infty), \eta_{\infty}^{\prime}$ is simply the solvent viscosity $\eta$. For microrheology the average mobility of a particle in the equilibrium suspension is just $1 / k_{B} T$ times the short-time self-diffusivity $D_{0}^{S}$, and hence the equivalent of the highfrequency dynamic viscosity is the inverse of the short-time self-diffusivity $\left(6 \pi a_{h} D_{0}^{s} / k_{B} T\right)^{-1}$. The microviscosity increment is thus $\Delta \eta^{\text {micro }}=\eta^{\text {micro }}(\mathrm{Pe})-\left(6 \pi a_{h} D_{0}^{s} / k_{B} T\right)^{-1}$, where $\eta^{\text {micro }}(P e)=F / 6 \pi a_{h} U$ from the Stokes drag law definition of the microviscosity. Like $\eta_{\infty}^{\prime}$, the short-time self-diffusivity $D_{0}^{s}$ is a purely hydrodynamic quantity and is simply given by the Stokes-Einstein-Sutherland value for an isolated particle in the absence of hydrodynamic interactions. As mentioned before, $D_{0}^{s} \times \eta_{\infty}^{\prime} \approx$ const, and therefore we may use $\eta_{\infty}^{\prime}$ or $1 / D_{0}^{s}$ interchangeably, and hence both viscosity increments can be written relative to $\eta_{\infty}^{\prime}$.

The high-frequency dynamic viscosity corresponds to that of an equilibrium (random) microstructure and expresses the fact that the particles are rigid and do not deform affinely with the flow. In macrorheology this "passive" resistance could be scaled out by simply replacing the solvent viscosity with the high-frequency dynamic viscosity-it was as if the two particles found themselves in a medium with effective viscosity $\eta_{\infty}^{\prime}$. The volume fraction dependence of all hydrodynamic functions were captured by this simple rescaling and the remaining volume fraction dependence came from structural deformations (e.g., at low or high Péclet), which could be estimated from the pair problem; hydrodynamics could be neglected completely for shear thinning, while for shear thickening only knowledge of the boundary layer was necessary. ${ }^{32}$ The structure of the microrheology problem suggests that this simple, but powerful, approximation should be applicable and we use it in what follows.

In the linear response regime, the average velocity corresponds to the long-time self-diffusivity, and thus the natural scale for the magnitude of the microviscosity is the inverse of the long-time self-diffusivity; specifically, $\eta^{\text {micro }} / \eta$ $=D_{a} / D_{\infty}^{s}$ as $P e \rightarrow 0$, where $D_{a}=k_{B} T / 6 \pi \eta a_{h}$ is the StokesEinstein-Sutherland diffusivity of an isolated particle. At small $P e$, the distortion of the microstructure occurs over the length scale of the probe-bath particle interaction, $\mathcal{O}(a+b)$, and the time scale for the microstructural response on this length scale is inversely proportional to the concentration- 
dependent long-time self-diffusivity $\tau \sim(a+b)^{2} / D_{\infty}^{s}(\phi)$. Thus, the appropriate Péclet number for the response is $P e$ $=U(a+b) / D_{\infty}^{r}(\phi)$, where $D_{\infty}^{r}(\phi)$ is the relative diffusivity (appropriate for fixed force or fixed velocity) at long times. (Technically, the fixed velocity problem does not correspond precisely to the long-time self-diffusivity as $P e \rightarrow 0$, but we do not expect a significant quantitative difference; the difference in the dilute no-hydrodynamic case is a factor of 2.) Now, in the case of fixed velocity the $U$ in the Péclet number is the actual velocity at which the particle is dragged. For fixed force, however, the velocity is not given but rather should be the average velocity $\langle U\rangle$ with which the probe moves, which is proportional to $D_{\infty}^{s} F / k_{B} T$, and therefore $P e_{F}=F(a+b) / k_{B} T \times D_{\infty}^{s} / D_{\infty}^{r}$. The ratio of the long-time selfdiffusivity to the long-time relative diffusivity depends on the size ratio between the probe and bath particles, but should not depend on the bath particle concentration. Thus, the constant force Péclet number should be independent of $\phi$. Hence, throughout the shear thinning regime - that is, up to $P e \sim \mathcal{O}(1)$ - both the fixed-force and fixed-velocity microviscosity scaled by the long-time self-diffusivity should be a universal function of the Péclet number based on the longtime self-diffusivity. Further, studies of the self-diffusivites ${ }^{36}$ suggest that the long-time self-diffusivity may be factored into the hydrodynamically determined short-time selfdiffusivity times the long-time self-diffusivity in the absence of hydrodynamic interactions: $D_{\infty}^{s} \approx D_{0}^{s} \times \hat{D}_{\infty}^{\text {nohydro }}$, and since $D_{0}^{s} \sim 1 / \eta_{\infty}^{\prime}$ we are able to scale out $\eta_{\infty}^{\prime}$ as in macrorheology, i.e., in magnitude $\Delta \eta^{\text {micro }} \sim \eta_{\infty}^{\prime} / \hat{D}_{\infty}^{\text {nohydro }}$.

At high Péclet numbers the situation is somewhat different, as is the case for macrorheology. Here, all the action is in the thin $\mathcal{O}((a+b) / P e)$ boundary layer at probe-bath particle contact. The response time is now the time to diffuse the thickness of the boundary layer $\tau \sim \delta^{2} / D$, where $\delta \sim(a$ $+b) / P e$ and the diffusivity is that for diffusion over this scale, which is the short-time self-diffusivity $D_{0}^{s}$. Thus, the appropriate Péclet number is that based on the short-time self-diffusivity, rather than the long-time self-diffusivity which is applicable at low $P e$. For the fixed force case the velocity is again the average velocity we seek, $P e_{F}=\langle U\rangle(a$ $+b) / D_{0}^{r}(\phi)$, while for fixed velocity $P e_{U}=U(a+b) / D_{0}^{s}(\phi)$. From the expression for the average velocity in the fixed force problem (5) (or its counterpart in Appendix A with hydrodynamics) we see that the average velocity is proportional to the average mobility times the difference between the driving force and the reactive Brownian force. The average mobility is proportional to $1 / 6 \pi \eta_{\infty}^{\prime} a_{h}$, as the solvent viscosity can be replaced by the high-frequency dynamic viscosity. The reactive Brownian force is proportional to $\phi$ times the pair-distribution function at contact, which is of $\mathcal{O}(\mathrm{Pe})$ in amplitude times the value of the pair-distribution function just outside the boundary layer, which we denote as $g^{\infty}(1 ; \phi)$. Combining these estimates in (5) shows that at high-Péclet number the microviscosity increment scales as $\Delta \eta^{\text {micro }} \sim \eta_{\infty}^{\prime} \times \phi g^{\infty}(1 ; \phi)$ - the microviscosity scales as the average "resistivity", which is proportional to $\eta_{\infty}^{\prime}$, times the number of particles contacting the probe, $\phi g^{\infty}(1 ; \phi) .{ }^{42}$ The pair probability outside the boundary layer needs to be determined, but its magnitude with respect to $\phi$ should scale like the equilibrium pair-distribution function at contact, $g^{e q}(1 ; \phi)$, which, for equal probe and bath particle radii, can be found from the Carnhan-Starling equation of state for hard spheres. Note with this estimate for the magnitude of $\langle U\rangle$, we have $P e_{F} \sim F(a+b) / k_{B} T \times 1 /\left[1+C \phi g^{\mathrm{eq}}(1 ; \phi)\right]$, where $C$ is an $\mathcal{O}(1)$ constant.

To summarize, we expect that the shear thinning component of the microviscosity increment will scale like

$$
\Delta \eta^{\text {micro }} \sim \eta_{\infty}^{\prime}(\phi) \phi g^{\mathrm{eq}}(1 ; \phi) \frac{(1+\alpha)^{3}}{2} \frac{D_{a}}{D} V\left(P e^{*}\right),
$$

where $P e^{*}$ is a scaled Péclet number given for low $P e$ by

$$
\begin{aligned}
& P e_{U}^{*}=\frac{U(a+b)}{D_{\infty}^{s}(\phi)}, \\
& P e_{F}^{*}=\frac{F(a+b)}{k_{B} T} \frac{D_{\infty}^{s}}{D_{\infty}^{r}} \sim \frac{F(a+b)}{k_{B} T},
\end{aligned}
$$

and

$$
\begin{aligned}
& P e_{U}^{*}=\frac{U(a+b)}{D_{0}^{s}(\phi)}, \\
& P e_{F}^{*}=\frac{F(a+b)}{k_{B} T}\left[1+C \phi g^{\mathrm{eq}}(1 ; \phi)\right]^{-1},
\end{aligned}
$$

for high $P e$. The ratio $D_{a} / D$ in (68) is only used to indicate the scalings with the size ratio $\alpha$ in the fixed-force or fixedvelocity cases; all the volume-fraction dependence is accounted for by $\eta_{\infty}^{\prime}(\phi) \phi g^{\mathrm{eq}}(1 ; \phi)$ and in $P e^{*}$. In writing (68) for low Péclet number we have used the estimate that the long-time self-diffusivity in the absence of hydrodynamic interactions scales like $1 / \phi g^{e q}(1 ; \phi)$, which was shown to agree well with the available experimental data at least up to the glass transition at $\phi \approx 0.58 .^{36}$

The above arguments apply equally well whether or not there are hydrodynamic interactions between particles; only the values of the short- and long-time self-diffusivites are affected by hydrodynamics. Furthermore, the scaling estimate at high Péclet number also applies when forcethickening is present $(\lambda<1.1)$; in this case the function $V\left(P e^{*}\right)$ would, of course, be different. Also, the arguments used in arriving at this scaling estimate are based on ideas that may only be valid when the probe and bath particles are comparable in size-say $1 / 2 \leqslant \alpha \leqslant 2$ - outside this range other estimates may apply, and (68) should be used with caution.

As a final note, the above scaling estimate would only be expected to hold if the dispersion remains in a "liquid-like" state. At very high concentrations colloidal dispersions may become solidlike and display a yield stress. The above theory would not apply to such yielding systems. At high Péclet numbers the motion of the probe particle may be sufficient to locally "melt" the suspension, resulting in a finite viscosity and the above estimates would then apply. 


\section{DISCUSSION AND CONCLUSIONS}

In summary, the core of our approach involves determining the perturbation to the suspension microstructure caused by the motion of the probe. We have derived an equation for the microstructure and solved it in the simplest limiting case of hard-sphere interactions and negligible hydrodynamic interactions. This corresponds physically to systems in which extended interactions prevent the probe and bath particles from approaching close enough for their hydrodynamic interactions to be significant. While this limit may seem severe, it is surprisingly accurate for the analogous shear viscosity problem, ${ }^{30,31,33}$ in which case the shear thinning regime is (quantitatively) insensitive to hydrodynamic interactions. We find the viscosity increment, as measured using active microrheology, to force thin with $P e$, in a similar and almost identical fashion to macrorheologically measured behavior. Of course, neglecting hydrodynamic interactions means that the Einstein correction to the high-frequency dynamic viscosity $\left[\Delta \eta / \eta=2.5 \phi+\mathcal{O}\left(\phi^{2}\right)\right]$ does not appear. Our analysis specifically isolates the Brownian (microstructural) component, and our proposal to account for the Einstein (hydrodynamic) contribution follows the analogous strategy in the shear macrorheology problem.

Beneath the semi-quantitative agreement between the viscosity increments as measured by micro- and macrorheology (Fig. 7), however, lies an important property: the microstructural contribution to the microrheologically measured viscosity varies with $\phi$, whereas that for macrorheology varies with $\phi^{2}$. This difference arises because the former is sensitive to each collision between the probe and bath particles, whereas macrorheology involves collisions between bath colloids. If this scaling were not known, the two techniques would not appear to agree. The distinction between these results is thus both beneficial and detrimental. It belies the difference between the modes of measurement, and thus encodes additional physical information about the material; but complicates the understanding and analysis of more general materials for which such scalings are not known.

Another important distinction with the shear viscosity problem arises. In active microrheology, the probe and bath particles can have different sizes, whereas all particles are (typically) identical in the shear viscosity problem. We have examined $\Delta \eta$ for general probe/bath size ratio, and demonstrated that $\Delta \eta / \eta$ can diverge in the small-probe limit. In the large-probe limit, in which the probe acts like a "strainer," our results may have questionable applicability. The force range $a-a_{h}$ is a physically determined length scale that scales with, e.g., the length of grafted polymer "hairs" or the ionic screening length, meaning that $\lambda \rightarrow \infty$ may be difficult to attain for large probe particles.

Once the microstructural perturbation is known, many statistical quantities can be computed. We have calculated the effective viscosity increments and fluctuations about those values, for all values of $P e$ and general probe/bath size ratios, in the dilute limit. This results in a "universal" curve, onto which we expect a wide range of measurements will collapse. Furthermore, microstructural deformation fields can be measured and directly compared to theory. More gener- ally, the relation between microstructural deformations and probe forces [Eq. (5)] could be tested directly given sufficient experimental resolution. Any discrepancy would come from hydrodynamic interactions or non-hard-sphere interparticle interactions, and would thus provide an experimental handle for these effects.

We have proposed methods to modify this theory to account for less dilute systems. Comparisons with measurements and simulations will be necessary to determine whether these ideas are applicable in the active microrheology context.

It would be of interest to study active microrheology with hydrodynamic interactions $(1<\lambda<\infty)$, for several reasons. Incorporating hydrodynamic interactions will naturally capture the hydrodynamic contribution to viscosity, and give more reliable results in the large-probe limit. Furthermore, as with the macrorheology of sheared suspensions, lubrication interactions (in the limit $\lambda \rightarrow 1$ ) are expected to cause shear thickening. ${ }^{30}$ Recent work by Khair and Brady ${ }^{34}$ shows this to be the case.

An important result highlighted above is that the effective viscosity increment measured in active microrheology depends on whether the probe is pulled at constant velocity, with constant force, or with a mixture of the two. In macrorheology, this distinction would be analogous to differences between constant-stress and constant-shear rate modes, which we expect to be negligible (away from any critical points). In fact, a series of microrheological experiments at different $\mathrm{Pe}$ might switch from one mode to the other as $\mathrm{Pe}$ is increased, which would complicate or obfuscate interpretation. Care should thus be exercised in real experiments. A similar difference, albeit hydrodynamic in origin, was noted for falling-ball rheometry. ${ }^{37}$ Generally, the constant velocity mode seems to yield a higher effective viscosity increment than the constant force mode.

Unsteady effects present an obvious and important generalization to the present study. For example, experiments in which probe particles are oscillated with finite-amplitude (or force) would provide information about the "healing time" of the microstructure. A high- $\mathrm{Pe}$ oscillitory probe should carve out (and remained confined to) a void region, so long as the oscillation frequency is sufficiently high that the void does not have time to close. One would expect, however, a qualitative change in behavior below a crossover frequency, where bath colloids diffusively "heal" the void before the probe returns.

Another interesting unsteady system is the microrheological analog of so-called stress-jump experiments. ${ }^{5}$ As in macrorheology, these would allow experiments to differentiate between the hydrodynamic and Brownian contributions to the viscosity increment. In such experiments, a probe would be moved steadily (where it is subject to hydrodynamic and Brownian forces) and suddenly allowed to stop. The hydrodynamic component of $\Delta \eta$ would vanish immediately, leaving microstructural perturbations alone, whose (diffusive) relaxation could be directly measured.

In this article, we have analyzed the simplest "complex" fluid possible, for which much is known and a well-defined, well-posed mathematical treatment can be derived starting 
with very basic assumptions. On the other hand, most complex materials are not nearly so "simple," and it is of tremendous importance (but substantial difficulty) to generalize the present approach to treat more complicated microstructures. Filamentous actin is a favorite model bio-material of microrheologists (both passive $\mathrm{e}^{15,43,44}$ and active $\mathrm{e}^{22,23,25}$ ) that consists of a network of elastic fibers that can be cross-linked to varying degrees. A simple model system for such materials would require the elastic nature of the microstructural elements to be incorporated. The mathematical structure of this problem would seem to be related to the system discussed in Sec. VIII and Appendix A 3. We hope that the framework we have provided here will form a basis upon which to address these types of problems.

As discussed in the Introduction, we feel it is important to de-emphasize the requirement that microrheological measurements faithfully reproduce macrorheological ones. While such agreement would certainly facilitate analysis and understanding, disagreements would by no means render microrheology unsuitable as a technique. Quite the oppositeany disagreements would result from the physical difference between methods for probing the materials, and would in fact yield additional information about the material in question. While it would be convenient to use the set of tools developed for macrorheology to analyze microrheological data, one need not take measurements in the micro-world, and then employ techniques from the macro-world to say something once again about the micro-world. Since microrheology probes materials on different length scales than macrorheology, and since active microrheology probes materials in a physically different manner, models based on microrheology would seem more appropriate for obtaining micro-structural information from microrheological data.

We close with the general observation that nonequilibrium microstructural deformations play a significant role in the motion and forcing of probe particles. This article has focused on the interaction between a probe and the microstructural deformation it induces (the most relevant for active and nonlinear microrheology). When multiple probes are present, however, the microstructural perturbation established by one can affect the motion/force of the others, which can lead to attractive or repulsive forces between two or more probes and may result in interesting collective dynamics and pattern formation. Similarly, multiparticle microrheology may also allow normal stress differences to be measured microrheologically. We leave the rich world of multiparticle active microrheology and deformationmediated interactions for future work.

\section{ACKNOWLEDGMENTS}

T.M.S. wishes to acknowledge the support of an NSF Applied Mathematics Postdoctoral Fellowship and a Lee A. Dubridge Prize Postdoctoral Fellowship. Conversations with Eric Weeks, Eric Furst, Ileana Carpen and Aditya Khair are gratefully acknowledged.

\section{APPENDIX A: SYSTEMATIC DERIVATION}

We consider a viscous suspension of colloidal particles and denote the probability density for finding the $N$ particles in a given configuration $P_{N}\left(\boldsymbol{x}_{1}, \boldsymbol{x}_{2}, \ldots, \boldsymbol{x}_{N}, t\right)$, where label 1 refers to the probe particle and labels $2 \rightarrow N$ to the $N-1$ bath particles. The particles move under the action of an external force $\boldsymbol{F}^{\text {ext }}$, interparticle colloidal forces denoted as $\boldsymbol{F}^{P}$ and entropic or thermal forces $-k_{B} T \nabla \ln P_{N}$. The velocity of any particle $\xi$ is given by

$$
\boldsymbol{U}_{\xi}=\sum_{\beta=1}^{N} \boldsymbol{M}_{\xi \beta} \cdot\left(\boldsymbol{F}_{\beta}^{e x t}+\boldsymbol{F}_{\beta}^{P}-k_{B} T \nabla_{\beta} \ln P_{N}\right),
$$

where $\boldsymbol{M}_{\xi \beta}(\boldsymbol{x})$ is the configuration-dependent hydrodynamic mobility coupling the velocity of particle $\xi$ to the force exerted on particle $\beta$. In the absence of hydrodynamic interactions the mobility tensor is diagonal and constant: $\boldsymbol{M}_{\xi \beta}$ $=1 / 6 \pi \eta a_{\xi} I \delta_{\xi \beta}$.

For a statistically homogeneous suspension we refer all particles relative to the probe 1, defining $\boldsymbol{r}_{\xi}=\boldsymbol{x}_{\xi}-\boldsymbol{x}_{1}$ and thus the velocity of any particle $\xi$ can be written as

$$
\boldsymbol{U}_{\xi}=\sum_{\beta=1}^{N}\left[\boldsymbol{M}_{\xi \beta} \cdot\left(\boldsymbol{F}_{\beta}^{e x t}+\boldsymbol{F}_{\beta}^{P}\right)-\left(\boldsymbol{D}_{\xi \beta}-\boldsymbol{D}_{\xi 1}\right) \cdot \nabla_{\beta} \ln P_{N}\right],
$$

where the translational diffusion tensor is given by $\boldsymbol{D}_{\xi \beta}$ $=k_{B} T M_{\xi \beta}$, all derivatives are with respect to the relative coordinates $\boldsymbol{r}_{\beta}$, and the absolute position of the probe particle does not matter so derivatives with respect to $x_{1}$ are zero.

The average motion of the probe particle is obtained from (A2) by averaging over the positions of the $N-1$ bath particles. The $N$-particle probability distribution can be written as $P_{N}\left(\boldsymbol{x}_{1}, \boldsymbol{r}_{2}, \ldots, \boldsymbol{r}_{N}, t\right)$ $=P_{N-1 / 1}\left(\boldsymbol{r}_{2}, \boldsymbol{r}_{3}, \ldots, \boldsymbol{r}_{N}, t \mid \boldsymbol{x}_{1}, t\right) P_{1}\left(\boldsymbol{x}_{1}, t\right)$, where $P_{N-1 / 1}$ is the conditional probability for finding the $N-1$ bath particles in configuration $\boldsymbol{r}_{N-1}$ given that the probe particle is at location $\boldsymbol{x}_{1}$. Owing to the statistical homogeneity of the suspension, $P_{N-1 / 1}$ does not depend on the position of the probe. Thus, the average velocity of the probe is defined by

$$
\left\langle\boldsymbol{U}_{1}\right\rangle_{1} \equiv \int \boldsymbol{U}_{1} P_{N-1 / 1}\left(\boldsymbol{r}_{2}, \boldsymbol{r}_{3}, \ldots, \boldsymbol{r}_{N}, t\right) d \boldsymbol{r}_{2} \ldots d \boldsymbol{r}_{N}
$$

where the subscript 1 on \langle\rangle$_{1}$ refers to the conditional average relative to the probe particle at $\boldsymbol{x}_{1}$.

The cases for the fixed force, fixed velocity and mixed conditions are different and so we shall proceed with these separately.

\section{Fixed force}

A fixed force corresponds to a constant external force exerted on particle 1 only. Thus, substituting (A2) into (A3) we have 


$$
\begin{aligned}
\left\langle\boldsymbol{U}_{1}\right\rangle_{1}^{F} \equiv & \int \boldsymbol{U}_{1} P_{N-1 / 1}\left(\boldsymbol{r}_{2}, \boldsymbol{r}_{3}, \ldots, \boldsymbol{r}_{N}, t\right) d \boldsymbol{r}_{2} \ldots d \boldsymbol{r}_{N} \\
= & \left(\int \boldsymbol{M}_{11} P_{N-1 / 1} d \boldsymbol{r}_{N-1}\right) \cdot \boldsymbol{F}_{1}^{e x t}+\sum_{\beta=1}^{N} \int\left[\boldsymbol{M}_{1 \beta} \cdot \boldsymbol{F}_{\beta}^{P}\right. \\
& \left.-\left(\boldsymbol{D}_{1 \beta}-\boldsymbol{D}_{11}\right) \cdot \nabla_{\beta} \ln P_{N-1 / 1}\right] P_{N-1 / 1} d \boldsymbol{r}_{N-1} \\
= & \left\langle\boldsymbol{M}_{11}\right\rangle \cdot \boldsymbol{F}_{1}^{e x t}-\sum_{\beta=1}^{N} \int\left[\left(\boldsymbol{D}_{1 \beta}\right.\right. \\
& \left.\left.-\boldsymbol{D}_{11}\right) \cdot \nabla_{\beta} \ln \left(P_{N-1 / 1} / P_{N-1 / 1}^{e q}\right)\right] P_{N-1 / 1} d \boldsymbol{r}_{N-1}, \quad(\mathrm{~A} 4)
\end{aligned}
$$

where the superscript $F$ denotes fixed force. The first term on the right-hand side of (A4) is simply the average hydrodynamic mobility of the probe particle in the deformed microstructure. In obtaining the second term on the right-hand side (rhs) of (A4) we have used the fact that at equilibrium the Boltzmann distribution applies, with $P_{N}^{e q} \sim \exp \left(-V / k_{B} T\right)$ and $\boldsymbol{F}_{\beta}^{P}=-\nabla_{\beta} V$, to replace the interparticle forces with the derivative of the $\log$ of the equilibrium distribution, showing quite clearly that the entropic reactive force of the bath particles is only nonzero out of equilibrium. At equilibrium the second term on the rhs of (A4) is zero and the average velocity is equal to the equilibrium-averaged mobility times the external force $\left\langle\boldsymbol{U}_{1}\right\rangle_{1}^{F, e q}=\left\langle\boldsymbol{M}_{11}\right\rangle^{e q} \cdot \boldsymbol{F}_{1}^{e x t}$. The short-time self-diffusivity of the probe particle is simply equal to $k_{B} T$ times the average mobility: $\boldsymbol{D}_{0}^{s} \equiv k_{B} T\left\langle\boldsymbol{M}_{11}\right\rangle^{e q}$, which is related to the meansquare displacement of the probe particle in passive microrheology at short times. Here, short time means the displacements are small compared to the smallest length scale in the suspension, e.g., the bath or probe radius, interparticle separation, etc.

At longer times in passive microrheology one measures the linear response of the material corresponding to the deformation of the microstructure due to a weak external force. For a weak force the perturbation to the microstructure is linear in the force, and $P_{N-1 / 1}-P_{N-1 / 1}^{e q}$ is proportional to $\boldsymbol{F}_{1}^{e x t}$ via the Green's function of the Smolushowski equation (A8). Thus, $\left\langle\boldsymbol{U}_{1}\right\rangle_{1}^{F}$ is proportional to $\boldsymbol{F}_{1}^{e x t}$, with $k_{B} T$ times the proportionality tensor defining the time- or frequency-dependent self-diffusivity $\boldsymbol{D}^{s}(t)$. At short times $\boldsymbol{D}^{s}(t) \sim \boldsymbol{D}_{0}^{s}$, while at long times $\boldsymbol{D}^{s}(t) \sim \boldsymbol{D}_{\infty}^{s}$, the long-time self-diffusivity. It can be shown quite easily, as one would expect in the linearresponse regime, that the definition of $\left\langle\boldsymbol{U}_{1}\right\rangle_{1}^{F}$ and the microstructural evolution problem is identical to the problem of the self-diffusivity (at long wavelengths). ${ }^{36}$ Beyond the linear-response regime, the self-diffusion problem and the motion of an active probe are no longer so simply, if at all, related.

Equation (A4) for the average velocity of the probe applies quite generally for any form of interparticle colloidal forces, $\boldsymbol{F}^{P}$. Hard-sphere forces are special and allow a significant simplification, however. As first pointed out in Ref. 40 , at the $N$-particle level, hard-sphere forces are identically zero in the space of all accessible configurations; they only enter at the boundaries to enforce the no flux boundary con- dition. Thus, $\boldsymbol{F}_{\beta}^{P}$ in (A4) is identically zero leaving only the entropic force. A straightforward integration by parts in (A4) then yields

$$
\begin{aligned}
\left\langle\boldsymbol{U}_{1}\right\rangle_{1}^{F, H S}= & \left\langle\boldsymbol{M}_{11}\right\rangle \cdot \boldsymbol{F}_{1}^{e x t}+\sum_{\beta=1}^{N} \int\left[\nabla \cdot \left(\boldsymbol{D}_{1 \beta}\right.\right. \\
& \left.\left.-\boldsymbol{D}_{11}\right)\right] P_{N-1 / 1} d \boldsymbol{r}_{N-1}-\sum_{\beta=1}^{N} \int \oint \boldsymbol{n}_{\beta} \cdot\left(\boldsymbol{D}_{1 \beta}\right. \\
& \left.-\boldsymbol{D}_{11}\right) P_{N-1 / 1} d S_{\beta} d \boldsymbol{r}_{N-1 \neq \beta},
\end{aligned}
$$

where $d S_{\beta}$ is the "surface" available to particle $\beta$ and the integral, $d \boldsymbol{r}_{N-1 \neq \beta}$, is over the volume accessible to the $N-1$ $\neq \beta$ other particles. (The flux at infinity vanishes.) When the hard-sphere force is exerted at the hydrodynamic radius, i.e., full hydrodynamic interactions $(\lambda \equiv 1)$, the relative mobility of two particles at contact is zero and the surface integral in (A5) vanishes, leaving only the average of the divergence of the relative mobilities.

In the absence of hydrodynamic interactions $(\lambda \rightarrow \infty)$, which is the situation considered in the main sections of this paper, the opposite occurs: only $\boldsymbol{D}_{11}$ is nonzero and is a constant. Thus, the second term on the rhs of (A5) zeros and the average velocity becomes

$$
\left\langle\boldsymbol{U}_{1}\right\rangle_{1}^{F, H S}=\boldsymbol{M}_{11} \cdot\left[\boldsymbol{F}^{e x t}+n k_{B} T \oint \boldsymbol{n}_{2} g\left(\boldsymbol{r}_{2}, t\right) d S_{2}\right],
$$

where we have used the indistinguishability of the $N-1$ bath particles to integrate out over all but one particle located at $\boldsymbol{r}_{2}$ relative to the probe particle. In (A6) the number density of bath particles is $n=(N-1) / V$ and the pair-distribution function is defined by $n g\left(\boldsymbol{r}_{2}, t\right)=P_{1 / 1}\left(\boldsymbol{r}_{2}, t\right)$, with $P_{1 / 1}$ the conditional probability of finding a bath particle at $\boldsymbol{r}_{2}$ given the probe at the origin $\left(x_{1}\right)$. To obtain (5) in the text one needs to recognize that the normal $\boldsymbol{n}_{2}$ is out of particle 2, while the normal $\boldsymbol{n}$ in (5) is out of the probe particle. In the absence of hydrodynamic interactions the self-mobility is simply $\boldsymbol{M}_{11}$ $=1 / 6 \pi \eta a_{h} \boldsymbol{I}$, where $a_{h}$ is the hydrodynamic radius (not the excluded volume radius $a$ ) of the probe particle.

The probability density $P_{N}$ satisfies the Smoluchowksi equation

$$
\frac{\partial P_{N}}{\partial t}+\sum_{\xi=1}^{N} \nabla_{\xi} \cdot \dot{j}_{\xi}=0,
$$

where the flux of particle $\xi$ is simply $P_{N}$ times the velocity from (A1), $j_{\xi}=U_{\xi} P_{N}$. Switching to relative coordinates and noting that the absolute position of the probe particle does not matter (A7) becomes

$$
\frac{\partial P_{N-1 / 1}}{\partial t}+\sum_{\xi=1}^{N} \nabla_{\xi} \cdot\left(\boldsymbol{j}_{\xi}-j_{1}\right)=0,
$$

with the relative flux for fixed force given by

$$
\begin{aligned}
\left(\boldsymbol{j}_{\xi}-\boldsymbol{j}_{1}\right)^{F}= & {\left[\left(\boldsymbol{M}_{\xi 1}-\boldsymbol{M}_{11}\right) \cdot \boldsymbol{F}_{1}^{e x t}-\sum_{\beta=1}^{N}\left(\boldsymbol{D}_{\xi \beta}-\boldsymbol{D}_{1 \beta}-\boldsymbol{D}_{\xi 1}\right.\right.} \\
& \left.\left.+\boldsymbol{D}_{11}\right) \cdot \nabla_{\beta} \ln \left(P_{N-1 / 1} / P_{N-1 / 1}^{e q}\right)\right] P_{N-1 / 1} .
\end{aligned}
$$


To proceed further one integrates (A8) over the positions of $N-2$ bath particles to obtain

$$
\frac{\partial g}{\partial t}+\nabla_{2} \cdot\left\langle\boldsymbol{j}_{2}-\boldsymbol{j}_{1}\right\rangle_{2}=0
$$

where the conditional average of the flux is now with a bath particle at $\boldsymbol{r}_{2}$ and the probe particle at $\boldsymbol{x}_{1}$. The conditionally averaged relative flux is

$$
\begin{aligned}
\left\langle\boldsymbol{j}_{2}-\boldsymbol{j}_{1}\right\rangle_{2}^{F}= & {\left[\left\langle\boldsymbol{M}_{21}-\boldsymbol{M}_{11}\right\rangle_{2} \cdot \boldsymbol{F}_{1}^{e x t}-\left\langle\left(\boldsymbol{D}_{22}-\boldsymbol{D}_{12}-\boldsymbol{D}_{21}\right.\right.\right.} \\
& \left.\left.+\boldsymbol{D}_{11}\right) \cdot \nabla_{2} \ln \left(P_{N-1 / 1} / P_{N-1 / 1}^{e q}\right)\right\rangle_{2} \\
& -\int\left\langle\left(\boldsymbol{D}_{23}-\boldsymbol{D}_{13}-\boldsymbol{D}_{21}\right.\right. \\
& \left.\left.\left.+\boldsymbol{D}_{11}\right) \cdot \nabla_{3} \ln \left(P_{N-1 / 1} / P_{N-1 / 1}^{e q}\right)\right\rangle_{3} P_{1 / 2}\left(\boldsymbol{r}_{3} \mid \boldsymbol{r}_{2}\right) d \boldsymbol{r}_{3}\right] \\
& \times g\left(\boldsymbol{r}_{2}, t\right),
\end{aligned}
$$

which unfortunately cannot be written only in terms of $g\left(\boldsymbol{r}_{2}, t\right)$, but involves an infinite hierarchy of conditional averages with more and more particles fixed. A closure is sought by diluteness, neglecting the integral term on the rhs of (A11), breaking the conditional average of the relative diffusivities times the gradient of the log of the probabilities, and replacing $\ln \left(P_{N-1 / 1} / P_{N-1 / 1}^{e q}\right)$ with $\ln \left(g / g^{e q}\right)$, as all neglected terms are $\mathcal{O}(\phi)$, where $\phi=4 \pi b^{3} n / 3$ is the volume fraction of the bath particles. Thus, the dilute pair Smoluchowski equation becomes

$\frac{\partial g^{F}}{\partial t}+\nabla_{2} \cdot\left[\left(\boldsymbol{M}_{21}-\boldsymbol{M}_{11}\right) g^{F}\right] \cdot \boldsymbol{F}_{1}^{e x t}=\nabla_{2} \cdot g^{e q} \boldsymbol{D}_{r}^{F} \cdot \nabla_{2}\left(g^{F} / g^{e q}\right)$,

where we have defined the relative diffusivity

$$
\boldsymbol{D}_{r}^{F}=\boldsymbol{D}_{22}-\boldsymbol{D}_{12}-\boldsymbol{D}_{21}+\boldsymbol{D}_{11},
$$

and we have appended the superscript $F$ to remind us that $g^{F}$ corresponds to the microstructure for fixed force.

Again, for hard-sphere interparticle potentials the equilibrium pair-distribution function is everywhere zero in the accessible space and can be dropped from (A12).

In the absence of hydrodynamic interactions $\boldsymbol{D}_{r}^{F}=\left(D_{22}\right.$ $\left.+D_{11}\right) \boldsymbol{I} \equiv D_{F} \boldsymbol{I}=k_{B} T\left(a_{h}+b_{h}\right) / 6 \pi \eta a_{h} b_{h} \boldsymbol{I},\left(\boldsymbol{M}_{21}-\boldsymbol{M}_{11}\right) \cdot \boldsymbol{F}_{1}^{\text {ext }}=$ $-M_{11} \boldsymbol{F}_{1}^{\text {ext }}$, and (A12) is identical to (16) in the text.

The boundary conditions for the Smoluchowski equation are a random structure with large separations

$$
g^{F} \sim 1 \quad \text { as } \boldsymbol{r}_{2} \rightarrow \infty,
$$

and no relative flux at contact

$$
\boldsymbol{n}_{2} \cdot\left\langle\boldsymbol{j}_{2}-\boldsymbol{j}_{1}\right\rangle_{2}^{F}=0 \quad \text { at } r=a+b .
$$

\section{Fixed velocity}

The analysis for the case of a fixed velocity proceeds in the same manner as above and we shall be brief. For the fixed velocity $\boldsymbol{U}_{1}$ is given and $\boldsymbol{F}_{1}^{\text {ext }}$ is to be found. From the general expression for the velocity of a particle (A2), the velocity of particle 1 is

$$
\boldsymbol{U}_{1}=\boldsymbol{M}_{11} \cdot \boldsymbol{F}_{1}^{e x t}-\sum_{\beta=1}^{N}\left(\boldsymbol{D}_{1 \beta}-\boldsymbol{D}_{11}\right) \cdot \nabla_{\beta} \ln \left(P_{N-1 / 1} / P_{N-1 / 1}^{e q}\right),
$$

from which the external force is

$$
\begin{aligned}
\boldsymbol{F}_{1}^{e x t}= & \boldsymbol{M}_{11}^{-1} \cdot \boldsymbol{U}_{1}+\sum_{\beta=1}^{N}\left(\boldsymbol{M}_{11}^{-1}\right) \cdot\left(\boldsymbol{D}_{1 \beta}\right. \\
& \left.-\boldsymbol{D}_{11}\right) \cdot \nabla_{\beta} \ln \left(P_{N-1 / 1} / P_{N-1 / 1}^{e q}\right),
\end{aligned}
$$

where $\boldsymbol{M}_{11}^{-1}$ is the inverse of the mobility of the probe particle. Note that this mobility corresponds to a single particle moving under an applied force in a bath of force (and torque) free particles. Its inverse is not equal to the single particle resistivity $\boldsymbol{R}_{11}$, which corresponds to a single particle moving in a bath of fixed particles. The single particle resistivity $\boldsymbol{R}_{11}=\left[(\mathcal{M})^{-1}\right]_{11}$, where $\mathcal{M}$ stands for the grand mobility matrix composed of the individual particle-particle mobility matricies $\boldsymbol{M}_{\xi \beta}$ as blocks.

Averaging over the $N-1$ bath particles as before we have

$$
\begin{aligned}
\left\langle\boldsymbol{F}_{1}^{e x t}\right\rangle_{1}^{U}= & \left\langle\boldsymbol{M}_{11}^{-1}\right\rangle_{1} \cdot \boldsymbol{U}_{1}+\sum_{\beta=1}^{N} \int\left[( \boldsymbol { M } _ { 1 1 } ^ { - 1 } ) \cdot \left(\boldsymbol{D}_{1 \beta}\right.\right. \\
& \left.\left.-\boldsymbol{D}_{11}\right) \cdot \nabla_{\beta} \ln \left(P_{N-1 / 1} / P_{N-1 / 1}^{e q}\right)\right] P_{N-1 / 1} d \boldsymbol{r}_{N-1} .
\end{aligned}
$$

For hard-sphere interparticle forces $P_{N-1 / 1}^{e q}$ can be removed and an integration by parts performed to give the analog of (A5):

$$
\begin{aligned}
\left\langle\boldsymbol{F}_{1}^{e x t}\right\rangle_{1}^{U, H S}= & \left\langle\boldsymbol{M}_{11}^{-1}\right\rangle_{1} \cdot \boldsymbol{U}_{1}-\sum_{\beta=1}^{N} \int\left[\nabla \cdot ( \boldsymbol { M } _ { 1 1 } ^ { - 1 } ) \cdot \left(\boldsymbol{D}_{1 \beta}\right.\right. \\
& \left.\left.-\boldsymbol{D}_{11}\right)\right] P_{N-1 / 1} d \boldsymbol{r}_{N-1} \\
& +\sum_{\beta=1}^{N} \int \oint \boldsymbol{n}_{\beta} \cdot\left(\boldsymbol{M}_{11}^{-1}\right) \cdot\left(\boldsymbol{D}_{1 \beta}\right. \\
& \left.-\boldsymbol{D}_{11}\right) P_{N-1 / 1} d S_{\beta} d \boldsymbol{r}_{N-1 \neq \beta} .
\end{aligned}
$$

And in the absence of hydrodynamic interactions (A19) becomes

$$
\left\langle\boldsymbol{F}_{1}^{e x t}\right\rangle_{1}^{U, H S}=\left(\boldsymbol{M}_{11}^{-1}\right) \cdot \boldsymbol{U}_{1}-n k_{B} T \oint \boldsymbol{n}_{2} g\left(\boldsymbol{r}_{2}, t\right) d S_{2},
$$

which is identical to (A6) for the fixed-force case.

Although the expression for the average force in the absence of hydrodynamic interactions is identical to that for the average velocity, the Smoluchowski equations are not the same in the two cases. The general balance equations (A8) and (A10) are the same, but the relative flux of the particles is different and is given by 


$$
\begin{aligned}
\left(\boldsymbol{j}_{\xi}-\boldsymbol{j}_{1}\right)^{U}= & {\left[\left(\boldsymbol{M}_{\xi 1} \cdot \boldsymbol{M}_{11}^{-1}-\boldsymbol{I}\right) \cdot \boldsymbol{U}_{1}-\sum_{\beta=1}^{N}\left[\left(\boldsymbol{D}_{\xi \beta}-\boldsymbol{D}_{1 \beta}-\boldsymbol{D}_{\xi 1}\right.\right.\right.} \\
& \left.+\boldsymbol{D}_{11}\right)-\left(\boldsymbol{M}_{\xi 1} \cdot \boldsymbol{M}_{11}^{-1}-\boldsymbol{I}\right) \cdot\left(\boldsymbol{D}_{1 \beta}\right. \\
& \left.\left.\left.-\boldsymbol{D}_{11}\right)\right] \cdot \nabla_{\beta} \ln \left(P_{N-1 / 1} / P_{N-1 / 1}^{e q}\right)\right] P_{N-1 / 1} . \quad(\mathrm{A} 21)
\end{aligned}
$$

In the dilute limit for pair interactions only, the relative flux becomes

$$
\begin{aligned}
\left(\boldsymbol{j}_{2}-\boldsymbol{j}_{1}\right)^{U}= & \left(\boldsymbol{M}_{21} \cdot \boldsymbol{M}_{11}^{-1}-\boldsymbol{I}\right) \cdot \boldsymbol{U}_{1} g^{U}-\left[\boldsymbol{D}_{r}-\left(\boldsymbol{M}_{21} \cdot \boldsymbol{M}_{11}^{-1}\right.\right. \\
& \left.-\boldsymbol{I}) \cdot\left(\boldsymbol{D}_{12}-\boldsymbol{D}_{11}\right)\right] \cdot \nabla_{2} \ln \left(g^{U} / g^{e q}\right) g^{U} .
\end{aligned}
$$

And in the absence of hydrodynamic interactions (A22) becomes

$$
\left(\boldsymbol{j}_{2}-\boldsymbol{j}_{1}\right)^{U}=-\boldsymbol{U}_{1} g^{U}-\boldsymbol{D}_{22} \cdot\left[\nabla_{2} \ln \left(g^{U} / g^{e q}\right)\right] g^{U},
$$

showing quite clearly that only the bath particles are diffusing. The "relative" diffusivity now becomes $D_{r}=D_{U}=D_{22}$ $=k_{B} T / 6 \pi \eta b_{h}$, and the final Smoluchsowski equation and boundary conditions follow directly from the above expressions for the relative flux.

\section{Mixed mode}

In some active microrheology experiments neither the force nor the velocity is fixed, but rather the probe particle is trapped in an external potential well (e.g., optical or magnetic tweezers) and the well dragged through the suspension at a prescribed velocity $\boldsymbol{U}^{\text {trap }}$. If the well is very deep one would expect this situation to approximate the fixed velocity case. But how deep must the well be? Indeed, how does one actually impose a fixed velocity experimentally? For colloidal-sized probes one cannot attach, for example, a rigid rod, because a rod that would not disturb the microstructure surrounding the probe would need to be so small that it would be subject to thermal fluctuations and therefore would not be perfectly rigid. Thus, the analysis of this mixed mode is necessary for the interpretation of experimental data.

Since the external potential well now defines a specific origin in the system the statistical homogeneity of the system is broken and the above analyses need modification. The location of the trap, e.g., the minimum of the external potential, is denoted $\boldsymbol{x}_{0}(t)$ and moves with velocity $\boldsymbol{U}^{\text {trap }}(t)$ relative to the laboratory frame. The probability density for finding the probe at $\boldsymbol{x}_{1}$ and the $N-1$ bath particles at $\boldsymbol{x}_{\xi}, \xi \neq 1$, given that the external potential (trap) is at $x_{0}$ is denoted as $P_{N}\left(\boldsymbol{x}_{1}, \ldots, \boldsymbol{x}_{N} \mid \boldsymbol{x}_{0}, t\right)$. The velocity of any given particle $\xi$ in the lab frame is the same as in (A1) with the addition of the arbitrary uniform velocity of the laboratory frame $\boldsymbol{U}^{l a b}$. The corresponding flux of particle $\xi$ is just the velocity of particle $\xi$ times the $N$-particle probability density $P_{N}$, which satisfies the Smoluchowski equation (A7). The mean velocity of the probe measured in the lab frame is given by

$$
\left\langle\boldsymbol{U}_{1}\right\rangle_{0}=\int \boldsymbol{U}_{1} P_{N} d \boldsymbol{x}_{1} \ldots \boldsymbol{x}_{N},
$$

where the subscript " 0 " denotes that the trap is at $\boldsymbol{x}_{0}$.
It is most convenient for analysis and physical interpretation to first change to a coordinate system moving with the trap velocity $\boldsymbol{U}^{\text {trap }}$ and to measure positions relative to the trap. Thus, we define a new coordinate system relative to the trap $z_{\xi}=\boldsymbol{x}_{\xi}-\boldsymbol{x}_{0}(t)$, with $\boldsymbol{x}_{0}=\boldsymbol{X}_{0}+\boldsymbol{U}^{\text {trap }} t$, where $\boldsymbol{X}_{0}$ is the location of the trap at $t=0$. The spatial derivatives change according to $\nabla_{x_{\xi}}=\nabla_{z_{\xi}}, \xi \neq 0 ; \nabla_{x_{0}}=\nabla_{z_{0}}-\sum_{\xi=1}^{N} \nabla_{z_{\xi}}$ and the time derivative according to $\partial / \partial t=\partial / \partial t-\sum_{\xi=1}^{N} \boldsymbol{U}^{\text {trap }} \cdot \nabla_{z_{\xi}}$. Thus the Smoluchowski equation (A7) becomes

$$
\frac{\partial P_{N}}{\partial t}+\sum_{\xi=1}^{N} \nabla_{z_{\xi}} \cdot\left(j_{\xi}-U^{t r a p} P_{N}\right)=0,
$$

and the flux expressions remain unchanged in this frame. We now switch to a coordinate system where we measure the $N-1$ bath particles relative to the probe: $\boldsymbol{r}_{1}=z_{1} ; \boldsymbol{r}_{\xi}=z_{\xi}-z_{1}$, with $\nabla_{z_{\xi}}=\nabla_{\boldsymbol{r}_{\xi}}, \xi \neq 1 ; \nabla_{z_{1}}=\nabla_{\boldsymbol{r}_{1}}-\sum_{\beta=1}^{N} \nabla_{\boldsymbol{r}_{\beta}}$ to give

$$
\frac{\partial P_{N}}{\partial t}+\nabla_{r_{1}} \cdot\left(\boldsymbol{j}_{1}-\boldsymbol{U}^{\text {trap }} P_{N}\right)+\sum_{\xi=2}^{N} \nabla_{\boldsymbol{r}_{\xi}} \cdot\left(\boldsymbol{j}_{\xi}-\boldsymbol{j}_{1}\right)=0,
$$

and the flux of particle $\xi$ becomes

$$
\begin{aligned}
\boldsymbol{j}_{\xi}= & {\left[\boldsymbol{U}^{l a b}+\sum_{\beta=1}^{N} \boldsymbol{M}_{\xi \beta} \cdot\left(\boldsymbol{F}_{\beta}^{e x t}+\boldsymbol{F}_{\beta}^{P}\right)-\sum_{\beta=1}^{N}\left(\boldsymbol{D}_{\xi \beta}\right.\right.} \\
& \left.\left.-\boldsymbol{D}_{\xi 1}\right) \cdot \nabla_{\beta} \ln P_{N}-\boldsymbol{D}_{\xi 1} \cdot \nabla_{1} \ln P_{N}\right] P_{N} .
\end{aligned}
$$

And the average probe velocity (A24) now becomes an integration over $d \boldsymbol{r}_{1} \ldots d \boldsymbol{r}_{N}$.

The important difference for the mixed mode case compared with either the fixed force or fixed velocity cases is the explicit dependence on the position of the probe particle and the accompanying spatial derivatives with respect to the probe position. The simplest problem that involves the interaction of the probe with a single bath particle now has six degrees of freedom - the three coordinates of the bath particle relative to the probe $\left(\boldsymbol{r}_{2}\right)$ and the three coordinates of the probe relative to the trap $\left(\boldsymbol{r}_{1}\right)$. In the fixed force or velocity problems we needed only to consider the motion of the bath particle relative to the probe-three degrees of freedom. Also, the trap velocity enters explicitly into the Smoluchowski equation as the flux of the probe is relative to the "flux" of the trap $\left(\boldsymbol{U}^{\text {trap }} P_{N}\right)$.

At this point we have not specified any form for the trap force, $\boldsymbol{F}_{1}^{\text {ext }}$, although we have assumed that it does not depend on the origin of the laboratory frame. The only specification we shall make is that the external force does not depend on the configuration of the bath particles - the bath particles do not influence the trap-but it does depend on the location of the probe particle $\boldsymbol{F}_{1}^{\text {ext }}\left(\boldsymbol{r}_{1}, t\right)$. Note that $\boldsymbol{F}_{1}^{\text {ext }}, \boldsymbol{U}^{\infty}(t)$ and $\boldsymbol{U}^{l a b}(t)$ may be arbitrary functions of time.

The explicit appearance of the external force can be removed in the same manner by which the interparticle forces were replaced by the equilibrium distribution provided it is derivable from a potential. Define $P_{N}^{e q}$ to be the equilibrium distribution of the $N$ particles in the presence of the external 
potential when the external potential is not translating, $\boldsymbol{U}^{\text {trap }} \equiv 0$. (Technically, all that is required is that $\boldsymbol{U}^{\text {trap }}$ $-\boldsymbol{U}^{l a b}=0$.) Then without loss of generality

$$
\boldsymbol{F}_{\beta}^{e x t}+\boldsymbol{F}_{\beta}^{P}=-\nabla_{\beta}\left(V^{e x t}+V^{P}\right)=-k_{B} T \nabla_{\beta} \ln P_{N}^{e q},
$$

and the flux (A27) becomes

$$
\begin{aligned}
\boldsymbol{j}_{\xi}= & {\left[\boldsymbol{U}^{l a b}-\sum_{\beta=1}^{N}\left(\boldsymbol{D}_{\xi \beta}-\boldsymbol{D}_{\xi 1}\right) \cdot \nabla_{\beta} \ln \left(P_{N} / P_{N}^{e q}\right)\right.} \\
& \left.-\boldsymbol{D}_{\xi 1} \cdot \nabla_{1} \ln \left(P_{N} / P_{N}^{e q}\right)\right] P_{N} .
\end{aligned}
$$

Substituting (A29) into (A26) shows clearly that $P_{N}=P_{N}^{e q}$ provided that $\boldsymbol{U}^{l a b}=\boldsymbol{U}^{\text {trap }}$ and that the initial condition is the equilibrium distribution.

For a given external force or potential one now proceeds as in the fixed force or velocity cases by integrating over bath particles to derive a reduced set of hierarchical equations. In contrast to the prior cases, however, the lowest level problem is not the pair problem for the interaction between the probe and a bath particle, but the "single" problem of the probe interacting with the trap. This is most easily seen by ignoring the bath particles altogether $\left(\phi_{b} \rightarrow 0\right)$; the equation for the probe then becomes

$$
\frac{\partial P_{1}}{\partial t}+\nabla_{1} \cdot\left[\boldsymbol{U}^{l a b}-\boldsymbol{U}^{\text {trap }}-\boldsymbol{D}_{11} \cdot \nabla_{1} \ln \left(P_{1} / P_{1}^{e q}\right)\right] P_{1}=0
$$

where the subscript " 1 " denotes the probe particle. When the trap and the laboratory move at the same speed, the probe is in equilibrium with the trap potential and $P_{1}=P_{1}^{e q}$ $\sim \exp \left(-V^{e x t} / k T\right)$. When there is relative velocity between the trap and the laboratory frame the probe is driven out of equilibrium; however, at steady state the solution is the simple exponential $P_{1}=P_{1}^{e q} \exp \left(\boldsymbol{D}_{11}^{-1} \cdot \boldsymbol{U}^{r e l} \cdot \boldsymbol{r}_{1}\right)$, where $\quad \boldsymbol{U}^{r e l}=\boldsymbol{U}^{\text {lab }}$ $-\boldsymbol{U}^{\text {trap }}$, and the average velocity of the probe follows as $\left\langle\boldsymbol{U}_{1}\right\rangle_{0}=\boldsymbol{U}^{\text {trap }}$ : the probe is simply dragged along with the trap, although it is not located on average at the bottom of the trap $\boldsymbol{r}_{1}=0$.

The pair-problem for the interaction of the probe with a bath particle (and also with the trap) can be constructed from the general $N$-particle equation (A26). Here we write down the simplest problem for the joint probability $P_{2}\left(\boldsymbol{r}_{1}, \boldsymbol{r}_{2}\right)$ $=P_{1}\left(\boldsymbol{r}_{1}\right) g\left(\boldsymbol{r}_{2} \mid \boldsymbol{r}_{1}\right)$ for hard spheres neglecting all hydrodynamic interactions:

$$
\begin{aligned}
\frac{\partial P_{2}}{\partial t} & +\nabla_{1} \cdot \boldsymbol{U}^{r e l} P_{2}-D_{1} \nabla_{1}^{2} P_{2}-\left(D_{1}+D_{2}\right) \nabla_{2}^{2} P_{2} \\
& -M_{1} \boldsymbol{F}_{1}^{\text {ext }} \cdot \nabla_{2} P_{2}+2 D_{1} \nabla_{1} \cdot \nabla_{2} P_{2}=0,
\end{aligned}
$$

subject to the boundary conditions of no relative flux at the surface of contact between the probe and bath particles

$$
\begin{aligned}
\hat{\boldsymbol{r}}_{2} \cdot\left(\boldsymbol{j}_{2}-\boldsymbol{j}_{1}\right)= & \hat{\boldsymbol{r}}_{2} \cdot\left[\left(D_{1}+D_{2}\right) \nabla_{2} P_{2}-M_{1} \boldsymbol{F}_{1}^{\text {ext }} P_{2}\right] \\
& -\hat{\boldsymbol{r}}_{2} \cdot \nabla_{1} P_{2} D_{1}=0 \quad \text { at } r_{2}=a+b,
\end{aligned}
$$

and an undistorted structure far away

$$
P_{2} \sim n P_{1} \text { as } r_{2} \rightarrow \infty,
$$

where $P_{1}\left(\boldsymbol{r}_{1}\right)$ is the probability density for finding the probe at $\boldsymbol{r}_{1}$ and $n$ is the number density of bath particles. One recovers the fixed force problem when there is no dependence on the location of the probe $\boldsymbol{r}_{1}$.

\section{APPENDIX B: SOLUTION FOR ARBITRARY Pe}

Using the substitution

$$
g=1+e^{-P e z / 2} P e f,
$$

the advection-diffusion equataion (16) is transformed to the Helmholtz equation

$$
\nabla^{2} f=\kappa^{2} f
$$

where

$$
\kappa=\frac{P e}{2} .
$$

Here $f$ satisfies boundary conditions

$$
\begin{aligned}
& f(r \rightarrow \infty)=0, \\
& \left.f_{r}\right|_{r=1}+\left.\kappa \cos \theta f\right|_{1}=-\cos \theta e^{\kappa \cos \theta} .
\end{aligned}
$$

The general solution to the Helmholz equation (B2) is

$$
f(r, \theta)=\sum_{n=0}^{\infty} C_{n} h_{n}(\kappa r) P_{n}(\cos \theta),
$$

where $h_{n}(u)$ is given by

$$
h_{n}(u)=\frac{K_{n+1 / 2}(u)}{\sqrt{u}},
$$

and we have kept only the solutions that decay at infinity. The boundary condition at $r=1$ is satisfied when

$$
\begin{aligned}
\sum_{n=0}^{\infty} & \left(h_{n}^{\prime} C_{n}+h_{n+1} \frac{n+1}{2 n+3} C_{n+1}\right. \\
& \left.+h_{n-1} \frac{n}{2 n-1} C_{n-1}\right)_{u=\kappa} P_{n}(\cos \theta) \\
= & \sum_{n=0}^{\infty} b_{n}(\kappa) P_{n}(\cos \theta)
\end{aligned}
$$

where we have used the identity

$$
x P_{n}(x)=\frac{n}{2 n+1} P_{n-1}(x)+\frac{n+1}{2 n+1} P_{n+1}(x),
$$

and where the coefficients $b_{n}(\kappa)$ are given by

$$
b_{n}(\kappa)=-\frac{2 n+1}{2 \kappa} \int_{-1}^{1} x P_{n}(x) e^{\kappa x} d x .
$$

This represents a tridiagonal matrix problem, 


$$
\left(h_{n}^{\prime} C_{n}+h_{n+1} \frac{n+1}{2 n+3} C_{n+1}+h_{n-1} \frac{n}{2 n-1} C_{n-1}\right)_{u=\kappa}=b_{n},
$$

which can be inverted numerically or using a symbolic mathematics program.

Using (B1) and (B6) in (23), we obtain the relative increments

$$
\frac{\Delta \eta}{\eta}=\frac{3}{2}(\alpha+1)^{3} \phi \sum_{m=0}^{\infty} C_{m} h_{m}(\kappa) \int_{-1}^{1} x P_{m}(x) e^{-\kappa x} d x,
$$

which can be further simplified using (B10) to give

$$
\frac{\Delta \eta}{\eta}=-\frac{3}{2}(\alpha+1)^{3} \frac{D_{a}}{D} \phi \sum_{m=0}^{\infty} \frac{2}{2 m+1} C_{m} h_{m}(\kappa) b_{m}(-\kappa) \text {. }
$$

The first few terms thus obtained are

$$
\begin{aligned}
& C_{0} \sim \sqrt{\frac{2}{\pi}} \frac{\kappa^{2}}{2}\left(1+\frac{32}{45} \kappa^{2}-\frac{1}{3} \kappa^{3}+\frac{2171}{4725} \kappa^{4}\right), \\
& C_{1} \sim \sqrt{\frac{2}{\pi}} \frac{\kappa^{2}}{2}\left(1+\frac{14}{15} \kappa^{2}-\frac{1}{3} \kappa^{3}+\frac{821}{1575} \kappa^{4}\right), \\
& C_{2} \sim \sqrt{\frac{2}{\pi}} \frac{\kappa^{4}}{9}\left(1+\frac{71}{210} \kappa^{2}\right), \\
& C_{3} \sim \sqrt{\frac{2}{\pi}} \frac{\kappa^{6}}{150},
\end{aligned}
$$

giving an expression for $\mathrm{g}$, here given to $\mathcal{O}\left(P e^{5}\right)$,

$$
\begin{aligned}
g= & 1+\kappa e^{-\kappa r(1+\cos \theta)}\left[\kappa\left(1+\frac{32}{45} \kappa^{2}-\frac{\kappa^{3}}{3}\right) \frac{1}{r}+\left(1+\frac{14}{15} \kappa^{2}\right.\right. \\
& \left.-\frac{\kappa^{3}}{3}\right) \frac{1+\kappa r}{r^{2}} \cos \theta+\frac{2}{9} \kappa(1 \\
& \left.\left.+\frac{71}{210} \kappa^{2}\right) \frac{3+3 \kappa r+\kappa^{2} r^{2}}{r^{3}}\left(\frac{3 \cos ^{2} \theta-1}{2}\right)\right] .
\end{aligned}
$$

${ }^{1}$ R. G. Larson, The Structure and Rheology of Complex Fluids (Oxford University Press, New York, 1999).

${ }^{2}$ B. J. Maranzano and N. J. Wagner, "The effects of interparticle interactions and particle size on reversible shear thickening: Hard-sphere colloidal dispersions," J. Rheol. 45, 1205 (2001).

${ }^{3}$ J. Bender and N. J. Wagner, "Reversible shear thickening in monodisperse and bidisperse colloidal dispersions," J. Rheol. 40, 899 (1996).

${ }^{4}$ I. E. Zarraga, D. A. Hill, and D. T. Leighton, "The characterization of the total stress of concentrated suspensions of noncolloidal spheres in Newtonian fluids" J. Rheol. 44, 185 (2000).

${ }^{5}$ M. E. Mackay and B. Kaffashi, "Stress jumps of charged colloidal suspensions, measurement of the elastic-like and viscous-like stress components," J. Colloid Interface Sci. 174, 117 (1995).

${ }^{6}$ V. T. O'Brien and M. E. Mackay, "Stress components and shear thickening of concentrated hard sphere suspensions," Langmuir 16, 7931 (2000). ${ }^{7}$ H. Watanabe, M. L. Yao, A. Yamagishi, K. Osaki, T. Shitata, H. Niwa, and Y. Morishima, "Nonlinear rheological behavior of a concentrated spherical silica suspension," Rheol. Acta 35, 433 (1996).

${ }^{8}$ H. Watanabe, M. L. Yao, K. Osaki, T. Shikata, H. Niwa, and Y. Morishima, "Nonlinear rheology of a concentrated spherical silica suspension. 2. Role of strain in shear thickening." Rheol. Acta 36, 524 (1997).
${ }^{9}$ H. Watanabe, M. L. Yao, K. Osaki, T. Shikata, H. Niwa, and Y. Morishima, "Nonlinear rheology of concentrated spherical silica suspensions: 3. Concentration dependence," Rheol. Acta 38, 2 (1999).

${ }^{10}$ F. C. MacKintosh and C. F. Schmidt, "Microrheology," Curr. Opin. Colloid Interface Sci. 4, 300 (1999).

${ }^{11}$ T. Gisler and D. A. Weitz, "Tracer microrheology in complex fluids," Curr. Opin. Colloid Interface Sci. 3, 586 (1998).

${ }^{12}$ T. G. Mason and D. A. Weitz, "Optical measurements of frequencydependent linear viscoelastic moduli of complex fluids," Phys. Rev. Lett. 74, 1250 (1995).

${ }^{13}$ F. Gittes, B. Schnurr, P. D. Olmsted, F. C. MacKintosh, and C. F. Schmidt, "Microscopic viscoelasticity: Shear moduli of soft materials determined from thermal fluctuations," Phys. Rev. Lett. 79, 3286 (1997).

${ }^{14}$ A. J. Levine and T. C. Lubensky, "One- and two-particle microrheology," Phys. Rev. Lett. 85, 1774 (2000).

${ }^{15}$ J. C. Crocker, M. T. Valentine, E. R. Weeks, T. Gisler, P. D. Kaplan, A. G. Yodh, and D. A. Weitz, "Two-point microrheology of inhomogeneous soft materials," Phys. Rev. Lett. 85, 888 (2000).

${ }^{16}$ Sutherland's name has historically not appeared in connection with this relation. However, P. Hanggi has raised our awareness of a remarkable and little-known paper of Sutherland (Ref. 45), in which he derives what is now called the "Stokes-Einstein relation" in a clear and compelling fashion. Since Sutherland's work appeared in the same year, 1905, as Einstein's celebrated paper (Ref. 46) on Brownian motion, it seems only appropriate to append Sutherland's name to this fundamental relation.

${ }^{17}$ A. J. Levine and T. C. Lubensky, "Response function of a sphere in a viscoelastic two-fluid medium," Phys. Rev. E 63, 041510 (2001).

${ }^{18}$ J. L. McGrath, J. H. Hartwig, and S. C. Kuo, "The mechanics of f-actin microenvironments depend on the chemistry of probing surfaces," Biophys. J. 793258 (2000).

${ }^{19}$ B. S. Chae and E. M. Furst, "Probe surface chemistry dependence and local polymer network structure in f-actin microrheology," Langmuir 21 3084 (2005).

${ }^{20}$ P. Habdas, D. Schaar, A. C. Levitt, and E. R. Weeks, "Forced motion of a probe particle near the colloidal glass transition," Europhys. Lett. 67, 477 (2004).

${ }^{21}$ A. R. Bausch, W. Moller, and E. Sackmann, "Measurement of local viscoelasticity and forces in living cells by magnetic tweezers," Biophys. J. 76, 573 (1999).

${ }^{22}$ K. S. Zaner and P. A. Valberg, "Viscoelasticity of f-actin measured with magnetic microparticles," J. Cell Biol. 1092233 (1989).

${ }^{23}$ F. Ziemann, J. Radler, and E. Sackmann, "Local measurements of viscoelastic moduli of entangled actin networks using an oscillating magnetic bead micro-rheometer," Biophys. J. 66, 2210 (1994).

${ }^{24}$ F. G. Schmidt, F. Ziemann, and E. Sackmann, "Shear field mapping in actin networks by using magnetic tweezers," Eur. Biophys. J. 24, 348 (1996).

${ }^{25}$ F. Amblard, A. C. Maggs, B. Yurke, A. N. Pargellis, and S. Leibler, "Subdiffusion and anomalous local viscoelasticity in actin networks," Phys. Rev. Lett. 77, 4470 (1996).

${ }^{26}$ E. Helfer, S. Harlepp, L. Bourdieu, J. Robert, F. C. MacKintosh, and D. Chatenay, "Microrheology of biopolymer-membrane complexes," Phys. Rev. Lett. 85, 457 (2000).

${ }^{27}$ E. M. Furst, "Interactions, structure, and microscopic response: Complex fluid rheology using laser tweezers," Soft Mater. 1, 167 (2003).

${ }^{28}$ W. B. Russel, D. A. Saville, and W. R. Schowalter, Colloidal Dispersions (Cambridge University Press, Cambridge, 1989).

${ }^{29}$ J. F. Brady and J. F. Morris, "Microstructure of strongly sheared suspensions and its impact on rheology and diffusion," J. Fluid Mech. 348, 103 (1997).

${ }^{30}$ J. Bergenholtz, J. F. Brady, and M. Vicic, "The non-Newtonian rheology of dilute colloidal suspensions," J. Fluid Mech. 456, 239 (2002).

${ }^{31}$ D. R. Foss and J. F. Brady, "Brownian dynamics simulation of hard-sphere colloidal dispersions," J. Rheol. 44, 629 (2000).

${ }^{32}$ D. R. Foss and J. F. Brady, "Structure, diffusion and rheology of Brownian suspensions by Stokesian dynamics simulation," J. Fluid Mech. 407, 167 (2000).

${ }^{33}$ J. F. Morris and J. F. Brady, "Self-diffusion in sheared suspensions," J. Fluid Mech. 312, 223 (1996).

${ }^{34}$ Recently, A. Khair and J. F. Brady have investigated a related problem in active microrheology, calculating the microstructural perturbations and viscosity increments for a colloidal suspension $(a=b)$, accounting for hydrodynamic interactions $(1<\lambda<\infty)$. As in the macro-rheological case, shear (or force-) thickening occurs for $P e \gtrsim 1$ when lubrication interac- 
tions become significant $(\lambda-1 \ll 1)$, but only minor quantitative differences occur for larger $\lambda$.

${ }^{35}$ W. J. Frith, P. dHaene, R. Buscall, and J. Mewis, "Shear thickening in model suspensions of sterically stabilized particles," J. Rheol. 40, 531 (1996).

${ }^{36}$ J. F. Brady, "The long-time self-diffusivity in concentrated colloidal dispersions," J. Fluid Mech. 272, 109 (1994).

${ }^{37}$ Y. Almog and H. Brenner, "Non-continuum anomalies in the apparent viscosity experienced by a test sphere moving through an otherwise quiescent suspension," Phys. Fluids 9, 16 (1997).

${ }^{38}$ We have neglected the contribution from the high- $P e$ cylindrical shell "wake" trailing the probe, because it is $\mathcal{O}\left(P e^{-1}\right)$. The gradient in the wake remains $\mathcal{O}\left(P e^{2}\right)$ only so long as it retains a thickness of order $\delta \sim(a$ $+b) / P e$. The wake diffuses a distance $\delta$ over a time $\tau_{D} \sim \delta^{2} / D$, which occurs at a downstream distance $L \sim U \tau_{D} \sim \delta$. The integrand is thus $\mathcal{O}\left(P e^{3}\right)$ only over a region of volume $2 \pi(a+b) L \delta$, giving an integral of order $\mathcal{O}(P e)$ and an overall wake contribution of order $\mathcal{O}\left(P e^{-1}\right)$.

${ }^{39}$ J. C. van der Werff, C. G. de Kruif, C. Blom, and J. Mellema, "Linear viscoelastic behavior of dense hard-sphere dispersions," Phys. Rev. A 39, 795 (1989)

${ }^{40}$ J. F. Brady, "The rheological behavior of concentrated colloidal dispersions," J. Chem. Phys. 99, 567 (1993).
${ }^{41}$ J. F. Brady, "Computer simulation of viscous suspensions," Chem. Eng. Sci. 56, 2921 (2001).

${ }^{42}$ Such a simple two-particle argument can be used at high Péclet number because all the action is in the boundary layer at probe-bath particle contact, which is indeed a pair problem. At low Péclet number where the microstructure is disturbed over regions the size of the probe and bath particles, multiparticle interactions must be properly accounted for in any "effective" pair problem, which is a much more difficult undertaking. Fortunately, however, we know that the low-Pe limit must behave as the long-time self-diffusivity, from which scaling estimates can be obtained.

${ }^{43}$ M. L. Gardel, M. T. Valentine, J. C. Crocker, A. R. Bausch, and D. A. Weitz, "Microrheology of entangled f-actin solutions," Phys. Rev. Lett. 91, 158302 (2003).

${ }^{44}$ T. G. Mason, T. Gisler, K. Kroy, E. Frey, and D. A. Weitz, "Rheology of f-actin solutions determined from thermally driven tracer motion," J. Rheol. 44, 917 (2000).

${ }^{45} \mathrm{~W}$. Sutherland, "A dynamical theory of diffusion for non-electrolytes and the molecular mass of albumin," Philos. Mag. 9, 781 (1905).

${ }^{46}$ A. Einstein, "Uber die von der molekularkinetischen theorie der warme geforderte bewegung von in ruhenden flussigkeiten suspenderten teilchen," Ann. Phys. 17, 549 (1905). 